\title{
Navier-Stokes equations with first order boundary conditions
}

\author{
Steiger, Olivier
}

\begin{abstract}
On the basis of semigroup and interpolation-extrapolation techniques we derive existence and uniqueness results for the Navier-Stokes equations. In contrast to many other papers devoted to this topic, we do not complement these equations with the classical Dirichlet (no-slip) condition, but instead consider stress-free or slip boundary conditions. We also study various regularity properties of the solutions obtained and provide conditions for global existence
\end{abstract}

DOI: https://doi.org/10.1007/s00021-005-0184-4

Posted at the Zurich Open Repository and Archive, University of Zurich

ZORA URL: https://doi.org/10.5167/uzh-156393

Journal Article

Published Version

Originally published at:

Steiger, Olivier (2006). Navier-Stokes equations with first order boundary conditions. Journal of mathematical fluid mechanics, 8(4):456-481.

DOI: https://doi.org/10.1007/s00021-005-0184-4 


\title{
Navier-Stokes Equations with First Order Boundary Condi- tions
}

Olivier Steiger

Communicated by H. Amann

\begin{abstract}
On the basis of semigroup and interpolation-extrapolation techniques we derive existence and uniqueness results for the Navier-Stokes equations. In contrast to many other papers devoted to this topic, we do not complement these equations with the classical Dirichlet (no-slip) condition, but instead consider stress-free or slip boundary conditions. We also study various regularity properties of the solutions obtained and provide conditions for global existence.
\end{abstract}

Mathematics Subject Classification (2000). 35Q30, 76D05, 35K55.

Keywords. Navier-Stokes equations, stress-free and slip boundary conditions.

\section{Introduction}

Throughout this paper we suppose that $n \geq 2$ and that $\Omega$ is a bounded domain in $\mathbb{R}^{n}$ with smooth boundary, $\partial \Omega$, lying locally on one side of $\Omega$. We consider the nonstationary Navier-Stokes equations

$$
\left.\begin{array}{r}
\partial_{t} u+(u \cdot \nabla) u-\mu \Delta u+\nabla \pi=f \\
\operatorname{div}(u)=0
\end{array}\right\} \quad \text { in } \Omega \times(0, \infty)
$$

describing the motion of a viscous incompressible (isothermal) Newtonian fluid in $\Omega$. Here $f$ is a given exterior force field, and $u=\left(u^{1}, \ldots, u^{n}\right)$ and $\pi$ are the unknown velocity and pressure field, respectively. Moreover, $\mu>0$ is a given constant, the kinematic viscosity, and the (constant) density has been normalised to 1 .

An enormous amount of literature by numerous authors has been devoted to the study of these equations. However, the overwhelming majority of those works is concerned with the Navier-Stokes equations coupled with Dirichlet (no slip) boundary conditions, that is, the fluid is required to be at rest at the boundary $\partial \Omega$. It is the aim of this paper to derive solvability results for the Navier-Stokes equations with different, but nonetheless physically reasonable, boundary condi- 
tions.

We denote the stress tensor of the fluid by $T(u, \pi):=2 \mu D(u)-\left(\pi \delta_{i j}\right)_{i, j=1, \ldots, n}$, where $D(u):=\frac{1}{2}\left(\partial_{i} u^{j}+\partial_{j} u^{i}\right)_{i, j=1, \ldots, n}$ is the rate of deformation tensor and $\delta_{i j}$ is the Kronecker delta symbol. Then writing $\gamma$ for the trace operator, $\nu$ for the outward unit normal on $\partial \Omega$, and $\phi_{\nu}$ and $\phi_{\tau}$ for the normal and tangential components of $\phi$ on $\partial \Omega$, we want to consider the boundary conditions

$$
\mathcal{T}_{1}(u, \pi):=\gamma T(u, \pi) \nu=0 \quad \text { on } \partial \Omega \times(0, \infty),
$$

as well as

$$
\mathcal{T}_{2}(u, \pi):=(\gamma D(u) \nu)_{\tau}+(\gamma u)_{\nu} \nu=0 \quad \text { on } \partial \Omega \times(0, \infty) .
$$

The first boundary condition means that the normal surface stress of the fluid has to vanish. In the literature this condition is, therefore, usually called the stressfree boundary condition, and the system (1) and $(2)_{1}$ is referred to as the second boundary value problem for the Navier-Stokes equations, see e.g. [19].

The other boundary condition $(2)_{2}$ admits motion of the fluid at the boundary in tangential directions. It is often called the slip condition, cf. [9], [10].

Complementing equations (1) and $(2)_{k}$ with an initial condition for the velocity field, we arrive at the following initial boundary value problem to be studied

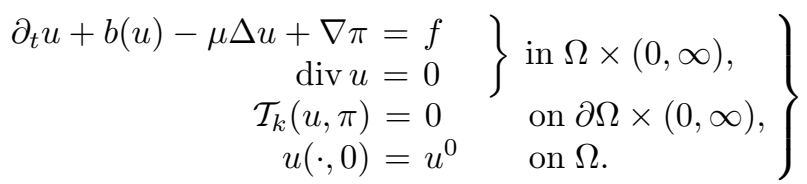

For abbreviation, we have set $b(u, v):=\operatorname{div}(u \otimes v)$, as well as $b(u):=b(u, u)$. Observe that $b(u, v)=(u \cdot \nabla) v$ provided $\operatorname{div}(u)=0$.

In the case of the stress-free boundary conditions the pressure field is present not only in the equation of motion but also in the boundary condition. This causes additional difficulties, because one cannot simply apply a Helmholtz-type projection to the equation of motion to get rid of the pressure. Nevertheless, it has been shown by G. Grubb and V. A. Solonnikov, e.g. [17], how this boundary value problem can be reduced, by pseudodifferential techniques, to one involving the velocity only.

On the other hand, we want to treat the case of slip boundary conditions differently, much more like the Navier-Stokes equations with Dirichlet boundary conditions, see [3]. The main reasons for doing so are the following: First, this approach is more elegant than the one used for the stress-free problem, in the sense that we do not have to work with pseudodifferential operators, and secondly the incompressibility constraint, $\operatorname{div}(u)=0$, can be naturally incorporated into the domain of definition of the differential operator under consideration.

Let us introduce a few notations: Suppose that $E$ and $F$ are Banach spaces. Then, by $\mathcal{L}(E, F)$ we mean the Banach space of all bounded linear operators from $E$ into $F$, and $\mathcal{L}(E):=\mathcal{L}(E, E)$. Furthermore, $\mathcal{L} i s(E, F)$ consists of all topological 
linear isomorphisms from $E$ onto $F$, and $\mathcal{L}$ aut $(E):=\mathcal{L} i s(E, E)$. If $G$ is a third Banach space then $\mathcal{L}(E, F ; G)$ is the set of all continuous bilinear maps from $E \times F$ into $G$. We set $\mathcal{L}^{2}(E, F):=\mathcal{L}(E, E ; F)$.

If $E$ is a linear subspace of $F$ and the natural injection $i:[x \mapsto x]$ belongs to $\mathcal{L}(E, F)$, we write $E \hookrightarrow F$. If $E$ is also dense in $F$, we express this by writing

$E \stackrel{d}{\hookrightarrow} F$, in this case $(F, E)$ is said to be a densely injected Banach couple. We also write $E \doteq F$ if $E \hookrightarrow F$ and $F \hookrightarrow E$, that is, if $E$ equals $F$ except for equivalent norms.

Now suppose in addition that $X \subset \mathbb{R}^{n}$ is a smooth bounded manifold. Then we denote by $W_{p}^{s}(X, E), H_{p}^{s}(X, E)$, and $B_{p, q}^{s}(X, E)$, the usual Sobolev-Slobedeckii, Bessel potential, and Besov spaces, respectively. To shorten the notation we set $W_{p}^{s}(X):=W_{p}^{s}(X, \mathbb{R})$ etc. We also employ the following convention: If $\mathfrak{F}\left(\Omega, \mathbb{R}^{n}\right)$ is a vector space of $\mathbb{R}^{n}$-valued distributions on $\Omega$ then we simply denote it by $\mathfrak{F}$. For instance,

$$
W_{p}^{s}:=W_{p}^{s}\left(\Omega, \mathbb{R}^{n}\right), \quad H_{p}^{s}:=H_{p}^{s}\left(\Omega, \mathbb{R}^{n}\right), \quad \text { etc. }
$$

\subsection{Solution operators and projections}

We denote by $K_{D}: \varphi \mapsto u$ the solution operator for the semi-homogeneous Dirichlet problem for the Laplacian

$$
-\Delta u=0 \quad \text { in } \Omega, \quad \gamma u=\varphi \quad \text { on } \partial \Omega,
$$

and by $R_{D}: f \mapsto u$ the solution operator for the other semi-homogeneous Dirichlet problem

$$
-\Delta u=f \quad \text { in } \Omega, \quad \gamma u=0 \quad \text { on } \partial \Omega .
$$

We know from Theorem 3.11 in [13], in view of [17, Example 2.1] and [13, Example $3.14]$, that these operators enjoy the following continuity properties for $1<p<\infty$ and $1 \leq q<\infty$

$$
K_{D} \in \mathcal{L}\left(B_{p, p}^{s-1 / p}(\partial \Omega), H_{p}^{s}(\Omega)\right) \cap \mathcal{L}\left(B_{p, q}^{s-1 / p}(\partial \Omega), B_{p, q}^{s}(\Omega)\right), \quad s \in \mathbb{R},
$$

and

$$
R_{D} \in \mathcal{L}\left(H_{p}^{s-2}(\Omega), H_{p}^{s}(\Omega)\right) \cap \mathcal{L}\left(B_{p, q}^{s-2}(\Omega), B_{p, q}^{s}(\Omega)\right), \quad s>1 / p .
$$

On the other hand, the Neumann problem for the Laplacian

$$
-\Delta u=f \quad \text { in } \Omega, \quad \partial_{\nu} u=\varphi \quad \text { on } \partial \Omega,
$$

has a solution $u$, uniquely determined up to a constant, provided the given functions $f$ and $\varphi$ satisfy the condition

$$
\int_{\Omega} f d x-\int_{\partial \Omega} \varphi d \sigma=0 .
$$


We fix a solution $u$ by requiring that $\int_{\Omega} u d x=0$, and denote the corresponding solution operators for the semihomogeneous problems by $K_{N}: \varphi \mapsto u$ and $R_{N}: f \mapsto u$.

The solution operators, introduced above, allow us to treat the decomposition of the Lebesgue space $L_{p}$ into divergence free functions and gradients of $H_{p}^{1}(\Omega)$ functions in a rather elegant way. If we define

$$
P_{k}:= \begin{cases}i d+\nabla R_{D} \operatorname{div}, & \text { if } k=1, \\ \left(i d-\nabla K_{N} \gamma_{\nu}\right)\left(i d+\nabla R_{D} \operatorname{div}\right), & \text { if } k=2,\end{cases}
$$

then the calculus of pseudodifferential operators, [13, Theorem 3.11 and Example 3.14], guarantees that

$$
P_{k}=\left(P_{k}\right)^{2} \in \mathcal{L}\left(H_{p}^{s}\right) \cap \mathcal{L}\left(B_{p, q}^{s}\right), \quad 1<p, q<\infty, \quad s>-1+1 / p .
$$

Now, restricting the maps $P_{1}$ and $P_{2}$ to $L_{p}$ we get the following two distinct direct sum decompositions of $L_{p}$

$$
L_{p}=\operatorname{im}\left(P_{k} \mid L_{p}\right) \oplus \operatorname{ker}\left(P_{k} \mid L_{p}\right), \quad k=1,2 .
$$

It is worth noting that the operator $P_{2}$ coincides with the usual Helmholtz-projection on $L_{p}$, which turns up in the study of the Navier-Stokes equations with no-slip boundary conditions, i.e.

$$
\operatorname{im}\left(P_{2} \mid L_{p}\right)=\left\{u \in L_{p} ; \operatorname{div}(u)=0, \gamma_{\nu} u=0\right\}=: L_{p, \sigma}
$$

and

$$
\operatorname{ker}\left(P_{2} \mid L_{p}\right)=\left\{u=\nabla g ; g \in H_{p}^{1}(\Omega)\right\} .
$$

The other projection $P_{1}$ is quite similar. Namely, we have that

$$
\operatorname{im}\left(P_{1} \mid L_{p}\right)=\left\{u \in L_{p} ; \operatorname{div}(u)=0\right\},
$$

as well as

$$
\operatorname{ker}\left(P_{1} \mid L_{p}\right)=\left\{u=\nabla g ; g \in H_{p}^{1}(\Omega): \gamma g=0\right\} .
$$

It is important to observe that the projection $P_{2}$ can be rewritten as

$$
P_{2}=i d-\nabla\left(K_{N} \gamma_{\nu}-R_{N} \operatorname{div}\right) .
$$

Moreover, we deduce again from [13, Theorem 3.11] that

$$
K_{N} \gamma_{\nu}-R_{N} \operatorname{div} \in \mathcal{L}\left(H_{p}^{s}, H_{p}^{s+1}\right) \cap \mathcal{L}\left(B_{p, q}^{s}, B_{p, q}^{s+1}\right), \quad s>-1+1 / p,
$$

in virtue of [17, Theorem 2.6].

\section{Preliminary results}

\subsection{Reduction of the problems}

Let us now carry out the reduction of the initial boundary value problem $(N S E)_{1}$. We follow here the ideas of G. Grubb and V. A. Solonnikov [17]. Applying the 
divergence operator to the first line of $(N S E)_{1}$ and multiplying the boundary condition by the outward unit normal $\nu$, we obtain for each $t>0$ that

$$
\begin{array}{rlrl}
-\Delta \pi & =\operatorname{div}(b(u)-f) & & \text { in } \Omega, \\
\gamma \pi=2 \mu(\gamma D(u) \nu)_{\nu} & & \text { on } \partial \Omega .
\end{array}
$$

With the aid of the solution operators and projections introduced above, we can therefore express the pressure in terms of the velocity $u$ and the exterior force $f$, so that substituting $\pi$ in the equation $(N S E)_{1}$ yields

$$
\left.\begin{array}{rlrl}
\partial_{t} u+P_{1} b(u)-\mu \Delta u+G_{1}(u) & =P_{1} f & & \text { in } \Omega \times(0, \infty), \\
\mathcal{B}_{1}(u) & =0 & & \text { on } \partial \Omega \times(0, \infty), \\
u(\cdot, 0) & =u^{0} & & \text { on } \Omega .
\end{array}\right\}
$$

Here, $G_{1}(u):=2 \mu \nabla K_{D}(\gamma D(u) \nu)_{\nu}$ is a singular Green operator (a non-local pseudodifferential operator) of second order and the trace operator $\mathcal{B}_{1}$ is defined by

$$
\mathcal{B}_{1}(u):=(\gamma D(u) \nu)_{\tau}+\gamma \operatorname{div}(u) \nu .
$$

The reason for being able to drop the condition $\operatorname{div}(u)=0$ in favour of $\gamma \operatorname{div}(u)=0$, stems from the fact that it can be recovered from (6), due to the unique solvability of the homogeneous heat equation with zero Dirichlet boundary conditions, see the proof of Theorem 3.8.

We set

$$
\boldsymbol{A}_{1} u:=\boldsymbol{A}_{1, p} u:=-\mu \Delta u+G_{1}(u), \quad u \in \operatorname{dom}\left(\boldsymbol{A}_{1}\right),
$$

with

$$
\operatorname{dom}\left(\boldsymbol{A}_{1}\right):=\left\{u \in H_{p}^{2} ; \mathcal{B}_{1}(u)=0\right\}=: H_{p, \mathcal{B}_{1}}^{2} .
$$

It follows from [14, Section 1.3], and likewise from [21] (whose approach is entirely different and does not rely on the calculus of pseudodifferential operators at all), that $\boldsymbol{A}_{1} \in \mathcal{L}\left(H_{p, \mathcal{B}_{1}}^{2}, L_{p}\right)$ is the negative infinitesimal generator of an analytic semigroup on $L_{p}$, which we indicate by

$$
\boldsymbol{A}_{1} \in \mathcal{H}\left(H_{p, \mathcal{B}_{1}}^{2}, L_{p}\right) .
$$

Concerning the dual operator of $\boldsymbol{A}_{1}$, considered as an unbounded linear operator in $L_{p}$, we can state the following Lemma.

Lemma 2.1. The dual operator of $\boldsymbol{A}_{1}$, in the sense of unbounded linear operators in $L_{p}$, is given by

$$
\boldsymbol{A}_{1}^{\prime} v=-\mu \Delta v, \quad v \in \operatorname{dom}\left(\boldsymbol{A}_{1}^{\prime}\right)
$$

with

$$
\operatorname{dom}\left(\boldsymbol{A}_{1}^{\prime}\right)=\left\{v \in H_{p^{\prime}}^{2} ; \mathcal{B}_{1}^{\prime}(v)=0\right\}=: H_{p^{\prime}, \mathcal{B}_{1}^{\prime}}^{2},
$$

where we have set

$$
\mathcal{B}_{1}^{\prime}(v)=S_{1}^{\prime}(v)+N_{1}^{\prime}(v),
$$


as well as

$$
\begin{aligned}
& S_{1}^{\prime}(v):=\partial_{\nu} v+\left(\kappa \nu-\operatorname{grad}_{\partial \Omega}\right) \gamma_{\nu} v-\left(s_{0}+\nu \operatorname{div} \partial \Omega\right) \gamma_{\tau} v, \\
& N_{1}^{\prime}(v):=2\left(\operatorname{grad}_{\partial \Omega}-\kappa \nu\right) \gamma_{\nu}\left(P_{1} v\right) .
\end{aligned}
$$

Here, $\operatorname{grad}_{\partial \Omega}$ and $\operatorname{div}_{\partial \Omega}$ denote the gradient and divergence operator on $\partial \Omega$, respectively, and $\kappa:=\gamma(\operatorname{div} \nu)$ stands for the mean curvature on $\partial \Omega$.

Proof. It is shown in [17] that the operator $A_{1}$ is elliptic and the corresponding trace operator $T_{1}$ is normal (in the sense of [12, Definition 1.4.3]). Moreover, according to [12, Lemma 1.6.8] the domain of definition of $A_{1}$ is dense in $L_{p}$. Hence, we can apply the general theory developed in [12] to derive an explicit characterisation of the dual operator of $A_{1}$. Interpreting the formulas in [12, Theorem 1.6.9] we immediately get that $A_{1}^{\prime}$ has the form stated in the assertion and that the corresponding trace operator $\mathcal{B}_{1}^{\prime}$ is normal. In particular, the nonlocal part $N_{1}^{\prime}$ of $\mathcal{B}_{1}^{\prime}$ is of class 0 .

We now turn our attention to the problem with slip-boundary conditions. In this case, applying the Helmholtz projection $P_{2}$ to the first line of $(N S E)_{2}$ eliminates the pressure from the equations.

$$
\left.\begin{array}{rlrl}
\partial_{t} u+P_{2} b(u)-\mu P_{2} \Delta u & =P_{2} f \\
\operatorname{div} u & =0 \\
\mathcal{B}_{2}(u) & =0 \\
u(\cdot, 0) & =u^{0}
\end{array} \quad \begin{array}{rl}
\text { on } \partial \Omega \times(0, \infty), \\
\text { on } \Omega .
\end{array}\right\}
$$

Here we have put

$$
\mathcal{B}_{2}(u):=(\gamma D(u) \nu)_{\tau}+(\gamma u)_{\nu} \nu
$$

This leads us to the following definition of the operator $\boldsymbol{A}_{2}$ and its domain of definition.

$$
\boldsymbol{A}_{2} u=-\mu P_{2} \Delta u, \quad u \in \operatorname{dom}\left(\boldsymbol{A}_{2}\right)
$$

with

$$
\operatorname{dom}\left(\boldsymbol{A}_{2}\right)=\left\{u \in H_{p}^{2} ; \mathcal{B}_{2}(u)=0, \operatorname{div}(u)=0\right\}=: H_{p, \mathcal{C}_{2}}^{2} .
$$

In [10] Y. Giga has studied the Navier-Stokes equations with general boundary conditions of first order and proven that, under an algebraic assumption on the coefficients of the boundary operator, the corresponding operator generates an analytic semigroup on $L_{p, \sigma}$, the space of solenoidal $L_{p}$-functions. As pointed out in [10] the slip boundary condition satisfies Giga's condition. Therefore,

$$
\boldsymbol{A}_{2} \in \mathcal{H}\left(H_{p, \mathcal{C}_{2}}^{2}, L_{p, \sigma}\right)
$$

Lemma 2.2. The operator $\boldsymbol{A}_{2}$ is formally self-adjoint, that is, the dual operator of $\boldsymbol{A}_{2}$, in the sense of unbounded linear operators in $L_{p, \sigma}$, is given by

$$
\boldsymbol{A}_{2}^{\prime} v=-\mu P_{2} \Delta v, \quad v \in \operatorname{dom}\left(\boldsymbol{A}_{2}^{\prime}\right)=H_{p^{\prime}, \mathcal{C}_{2}}^{2}
$$


Proof. Thanks to Gauss' theorem and the fact that the dual operator of $P_{2}$ considered as a bounded linear map in $L_{p}$ equals the canonical injection $i_{p^{\prime}}: L_{p^{\prime}, \sigma} \rightarrow L_{p^{\prime}}$, we obtain that

$$
\left\langle\boldsymbol{A}_{2} u, v\right\rangle=\left\langle u,-\mu P_{2} \Delta v\right\rangle, \quad u \in H_{p, \mathcal{C}_{2}}^{2}, \quad v \in H_{p^{\prime}, \mathcal{C}_{2}}^{2} .
$$

Since $\boldsymbol{A}_{2}$ and $\boldsymbol{A}_{2}^{\prime}$ are the negative generators of analytic semigroup it follows that their resolvent sets have a nonempty intersection. Now the assertion is obvious.

\subsection{Interpolation-extrapolation scales}

For the remainder of this paper we suppose that $1<p<\infty$ and that whenever $k$ appears as an index, it is assumed to belong to $\{1,2\}$.

Notations. Fix for each $\theta \in(0,1)$ an interpolation functor

$$
(\cdot, \cdot)_{\theta} \in\left\{[\cdot, \cdot]_{\theta},(\cdot, \cdot)_{\theta, p}\right\} .
$$

Here we have denoted by $[\cdot, \cdot]_{\theta}$ the complex, and by $(\cdot, \cdot)_{\theta, q}, 1<q<\infty$ the real interpolation functor of exponent $\theta \in(0,1)$ (and parameter $q$ ).

Now put

$$
F_{p}^{2 j}:=H_{p}^{2 j}, \quad j \in\{0, \pm 1\},
$$

and for $s=2 j+2 \theta$ with $j \in\{-1,0\}$ and $0<\theta<1$,

$$
F_{p}^{s}:= \begin{cases}H_{p}^{s}, & \text { if }(\cdot, \cdot)_{\theta}=[\cdot, \cdot]_{\theta}, \\ B_{p, p}^{s}, & \text { if }(\cdot, \cdot)_{\theta}=(\cdot, \cdot)_{\theta, p} .\end{cases}
$$

We also define the spaces whose elements satisfy the boundary conditions (denoted by $\mathcal{B}_{k}$ ), or all the additional conditions $\left(\mathcal{C}_{k}\right)$, in a suitable sense. Let us first assume that $k=1$, then

$$
F_{p, \mathcal{C}_{1}}^{s}:=F_{p, \mathcal{B}_{1}}^{s}:= \begin{cases}\left\{u \in F_{p}^{s} ; \mathcal{B}_{1}(u)=0\right\}, & 1+1 / p<s \leq 2, \\ F_{p}^{s}, & 0 \leq s<1+1 / p, \\ \left(F_{p^{\prime}, \mathcal{C}_{1}^{\prime}}^{-s}\right)^{\prime}, & -2 \leq s<0, \quad s \neq-1+1 / p,\end{cases}
$$

where $F_{p, \mathcal{C}_{1}^{\prime}}^{s}:=F_{p, \mathcal{B}_{1}^{\prime}}^{s}$ is defined for $0 \leq s \leq 2$ by replacing condition $\mathcal{B}_{1}(u)=0$ by $\mathcal{B}_{1}^{\prime}(u)=0$ in the above formula.

Now suppose that $k=2$, we set

$$
F_{p, \mathcal{B}_{2}}^{s}:= \begin{cases}\left\{u \in F_{p}^{s} ; \mathcal{B}_{2}(u)=0\right\}, & 1+1 / p<s \leq 2, \\ \left\{u \in F_{p}^{s} ; \gamma_{\nu} u=0\right\}, & 1 / p<s<1+1 / p, \\ F_{p}^{s}, & 0 \leq s<1 / p, \\ \left(F_{p^{\prime}, \mathcal{B}_{2}}^{-s}\right)^{\prime}, & -2 \leq s<0, \quad s \notin\{-2+1 / p,-1+1 / p\},\end{cases}
$$


as well as,

$$
F_{p, \mathcal{C}_{2}}^{s}:= \begin{cases}F_{p, \mathcal{B}_{2}}^{s} \cap L_{p, \sigma}, & 0 \leq s \leq 2, \quad s \notin\{1 / p, 1+1 / p\}, \\ \left(F_{p^{\prime}, \mathcal{C}_{2}}^{-s}\right)^{\prime}, & -2 \leq s<0, \quad s \notin\{-2+1 / p,-1+1 / p\} .\end{cases}
$$

To avoid bothersome distinction of cases, we also write $F_{p, \mathcal{B}_{2}^{\prime}}^{s}:=F_{p, \mathcal{B}_{2}}^{s}$.

Of course, we denote $F_{p, \mathcal{B}_{k}}^{s}$ and $F_{p, \mathcal{C}_{k}}^{s}$ by $H_{p, \mathcal{B}_{k}}^{s}$ and $H_{p, \mathcal{C}_{k}}^{s}$, respectively, if the underlying space is a Bessel potential space, and by $B_{p, p, \mathcal{B}_{k}}^{s}$ and $B_{p, p, \mathcal{C}_{k}}^{s}$, respectively, in the case of Besov spaces. Furthermore,

$$
L_{p, \mathcal{C}_{k}}:=F_{p, \mathcal{C}_{k}}^{0}= \begin{cases}L_{p}, & \text { if } k=1, \\ L_{p, \sigma}, & \text { if } k=2 .\end{cases}
$$

Remarks 2.3. (a) Observe that we have not defined the spaces $F_{p, \mathcal{B}_{k}}^{s}$ and $F_{p, \mathcal{C}_{k}}^{s}$ for all $s \in[-2,2]$, although this can be done in a consistent way, so that the assertions of the following lemma are true for all $s$. We refrain from doing this and refer the interested reader instead to [11], [20] and [23].

For the sake of convenience, let us define those exceptional values of $s$ depending on $1<p<\infty$ and $k \in\{1,2\}$

$$
\Sigma_{k, p}:= \begin{cases}\{-2+1 / p, 1+1 / p\}, & \text { if } k=1, \\ \{-2+1 / p,-1+1 / p, 1 / p, 1+1 / p\}, & \text { if } k=2 .\end{cases}
$$

(b) It follows immediately from the definitions that the spaces $F_{p, \mathcal{B}_{k}}^{s}$ coincide with the usual Bessel potential (or Besov) spaces for some $s \in[-2,2]$. Namely

$$
F_{p, \mathcal{B}_{1}}^{s}=F_{p}^{s}, \quad-1+1 / p<s<1+1 / p
$$

and

$$
F_{p, \mathcal{B}_{2}}^{s}=F_{p}^{s}, \quad-1+1 / p<s<1 / p .
$$

(c) The projection $P_{k}$ enjoys the following continuity properties

$$
P_{k} \in \mathcal{L}\left(F_{p, \mathcal{B}_{k}}^{s}\right) \cap \mathcal{L}\left(F_{p, \mathcal{B}_{k}}^{s}, F_{p, \mathcal{C}_{k}}^{s}\right), \quad-1+1 / p<s<1+1 / p,
$$

provided $s$ is not singular in the sense of remark (a).

Proof. Suppose that $-1+1 / p<s<1+1 / p$ with $s \notin \Sigma_{k, p}$. By the continuity of $P_{k}$ in $F_{p}^{s}$ and the fact that

$$
\operatorname{im}\left(P_{2} \mid F_{p}^{s}\right)=\left\{u \in F_{p}^{s} ; \operatorname{div}(u)=0, \gamma_{\nu} u=0\right\}, \quad s \geq 0,
$$

we infer that (9) is true if $k=1$ or if $k=2$ and $s \geq 0$. The assertion in the remaining cases follows by duality, confer also Section 2 in [6] (We note that the spaces called $\mathbb{H}_{p}^{s}$ there coincide with $H_{p, \mathcal{C}_{k}}^{s}$ for $-1+1 / p<s<1 / p$.)

(d) Let us consider the problem with slip boundary conditions, that is, $k=2$. Moreover, suppose that $-2 \leq s<0$ and $s \notin \Sigma_{2, p}$. Then we can show, by 
a modification of the proofs of [6, Lemma 2.1 and Theorem 2.2], that $F_{p, \mathcal{C}_{k}}^{s}$ is a closed subspace of $F_{p, \mathcal{B}_{k}}^{s}$ and that the map $P_{2} \mid L_{p}$ possesses a uniquely determined continuous extension $P_{2, s, p}$ on $F_{p, \mathcal{B}_{k}}^{s}$, such that $P_{2, s, p}$ is a projection of $F_{p, \mathcal{B}_{k}}^{s}$ onto $F_{p, \mathcal{C}_{k}}^{s}$. Of course, $P_{2, s, p}$ equals $P_{2} \mid F_{p, \mathcal{B}_{k}}^{s}$ if $-1+1 / p<s<0$ since $F_{p, \mathcal{B}_{k}}^{s}=F_{p}^{s}$ in this range.

(e) In this paper we restrict ourselves to the situation where $(\cdot, \cdot)_{\theta}$ is either the complex $[\cdot, \cdot]_{\theta}$ or the real interpolation functor $(\cdot, \cdot)_{\theta, p}$. However, it is not difficult to verify that all results remain valid if $(\cdot, \cdot)_{\theta}$ is chosen to be the real interpolation functor $(\cdot, \cdot)_{\theta, q}$ with $1<q<\infty$ arbitrary. This leads to interpolation-extrapolation scales which we obtain by setting $F_{p}^{s}:=B_{p, q}^{s}$ in the preceding definitions.

Lemma 2.4. Suppose that $0<\theta<1$ with $2 \theta \notin \Sigma_{k, p}$. Then

$$
\left(L_{p}, H_{p, \mathcal{B}_{k}}^{2}\right)_{\theta} \doteq F_{p, \mathcal{B}_{k}}^{2 \theta}, \quad \text { and } \quad\left(L_{p}, H_{p, \mathcal{B}_{k}^{\prime}}^{2}\right)_{\theta} \doteq F_{p, \mathcal{B}_{k}^{\prime}}^{2 \theta},
$$

as well as

$$
\left(L_{p, \mathcal{C}_{k}}, H_{p, \mathcal{C}_{k}}^{2}\right)_{\theta} \doteq F_{p, \mathcal{C}_{k}}^{2 \theta} .
$$

Proof. (a) Since both (system of) boundary operators $\mathcal{B}_{1}=(\gamma D(\cdot) \nu)_{\tau}+\gamma \operatorname{div}(\cdot) \nu$ and $\mathcal{B}_{2}=\left\{\left(\partial_{\nu} \cdot\right)_{\tau}, \gamma_{\nu}\right\}$ are normal (in the sense of Seeley), the first assertion follows from P. Grisvard [11], in the case of real interpolation, and from R. T. Seeley [20], for complex interpolation.

(b) Assume that $k=1$ and consider the operator $\mathcal{B}_{1}^{\prime}=S_{1}^{\prime}+N_{1}^{\prime}$. We set

$$
F_{p, S_{1}^{\prime}}^{s}:= \begin{cases}\left\{u \in F_{p}^{s} ; S_{1}^{\prime}(u)=0\right\}, & 1+1 / p<s \leq 2, \\ F_{p}^{s}, & 0 \leq s<1+1 / p .\end{cases}
$$

Then invoking again the results of Grisvard and Seeley for the normal trace operator $S_{1}^{\prime}$, we obtain that

$$
\left(L_{p}, H_{p, S_{1}^{\prime}}^{2}\right)_{\theta} \doteq F_{p, S_{1}^{\prime}}^{2 \theta}, \quad 0<\theta<1, \quad 2 \theta \neq 1+1 / p
$$

The boundary operator $\mathcal{B}_{1}^{\prime}$ contains the nonlocal parts $N_{1}^{\prime}$. Nevertheless, it is still possible to establish the asserted interpolation result by means of an isomorphism from $F_{p, \mathcal{B}_{1}^{\prime}}^{s}$ onto $F_{p, S_{1}^{\prime}}^{s}$

$$
\Lambda \in \mathcal{L} i s\left(F_{p, \mathcal{B}_{1}^{\prime}}^{s}, F_{p, S_{1}^{\prime}}^{s}\right), \quad 0 \leq s \leq 2, \quad s \neq 1+1 / p,
$$

see [12, Lemma 1.6.8]. The construction of $\Lambda$ relies on the fact that there exists an operator from $B_{p, p}^{s-1 / p}(\partial \Omega) \times B_{p, p}^{s-1-1 / p}(\partial \Omega)$ into $H_{p}^{s}(\Omega)$, which is a coretraction (a right inverse) of the trace operator $\rho=\left(\gamma, \partial_{\nu}\right)$ provided $s>1+1 / p$, and whose norm as a map from $B_{p, p}^{-1 / p}(\partial \Omega) \times B_{p, p}^{-1-1 / p}(\partial \Omega)$ to $L_{p}(\Omega)$ is arbitrarily small. The existence of such an operator is shown in Lemma 1.6.4 of [12] if $p=2$. In the non-Hilbert space setting one can construct such a map on the basis of the results in Appendix B of [5]. We omit the details of these constructions and refer 
to the articles already cited and to [22]. However, it is important to note, that the isomorphism $\Lambda$ does not depend on $1<p<\infty$.

(c) Now suppose that $k=2$. The last assertion follows from a simple modification of the proof of Lemma 3.2 in [3]. Note, in particular, that the operator defined by

$$
\boldsymbol{B} u:=-\mu \Delta u-\mu \nabla \operatorname{div}(u), \quad u \in \operatorname{dom}(\boldsymbol{B}):=H_{p, \mathcal{B}_{2}}^{2}
$$

is the negative generator of an analytic semigroup on $L_{p}$ and satisfies $P_{2} \boldsymbol{B} u=\boldsymbol{A}_{2} u$ for every $u \in \operatorname{dom}\left(\boldsymbol{A}_{2}\right)$.

We continue by proving an important embedding theorem. For this we recall that, given $0 \leq s_{0} \leq s_{1} \leq 2$ and $1<p_{0}, p_{1}<\infty$,

$$
F_{p_{1}}^{s_{1}} \hookrightarrow F_{p_{0}}^{s_{0}}, \quad s_{1}-n / p_{1} \geq s_{0}-n / p_{0} .
$$

The following theorem shows that an analogous results is true for the spaces $F_{p, \mathcal{B}_{k}}^{s}$ and $F_{p, \mathcal{C}_{k}}^{s}$.

Theorem 2.5. Suppose that $1<p_{0}, p_{1}<\infty$ and that $-2 \leq s_{0} \leq s_{1} \leq 2$ with $s_{j} \notin \Sigma_{k, p_{j}}$ for $j=0,1$. Moreover assume that

$$
s_{1}-n / p_{1} \geq s_{0}-n / p_{0} .
$$

Then

as well as

$$
F_{p_{1}, \mathcal{B}_{k}}^{s_{1}} \stackrel{d}{\hookrightarrow} F_{p_{0}, \mathcal{B}_{k}}^{s_{0}},
$$

$$
F_{p_{1}, \mathcal{C}_{k}}^{s_{1}} \stackrel{d}{\hookrightarrow} F_{p_{0}, \mathcal{C}_{k}}^{s_{0}} .
$$

Proof. First, we observe that the reflexivity of $L_{q}$ carries over to the spaces $F_{q, \mathcal{B}_{k}}^{s}$ and $F_{q, \mathcal{B}_{k}^{\prime}}^{s}$ for $1<q<\infty$ and $0 \leq s \leq 2$ with $s \notin \Sigma_{k, q}$.

(a) Now, suppose that $0 \leq s_{0} \leq s_{1} \leq 2$ and that condition (12) is satisfied. Then thanks to the embedding (11) and the definition of $F_{p_{j}, \mathcal{B}_{k}}^{s_{j}}$ we deduce that

$$
F_{p_{1}, \mathcal{B}_{k}}^{s_{1}} \hookrightarrow F_{p_{0}, \mathcal{B}_{k}}^{s_{0}} .
$$

Regarding the asserted density of the above embedding, let us define

$$
C_{\mathcal{B}_{k}}^{2}(\bar{\Omega})^{n}:=\left\{u \in C^{2}\left(\bar{\Omega}, \mathbb{R}^{n}\right) ; \mathcal{B}_{k}(u)=0\right\} .
$$

It is a consequence of the arguments of Section 5 in [4] (see also appendix B in [5]) that $C_{\mathcal{B}_{k}}^{2}(\bar{\Omega})^{n}$ is dense in $F_{q, \mathcal{B}_{k}}^{s}$, for $1<q<\infty$ and $s \in[0,2] \backslash \Sigma_{k, q}$. Therefore the embedding (13) is dense.

(b) Next, assume that $-2 \leq s_{0} \leq s_{1}<0$ and that (12) is fulfilled. Moreover, suppose that $k=1$, then similarly as in (a), we define the spaces

$$
C_{S_{1}^{\prime}}^{2}(\bar{\Omega})^{n}:=\left\{u \in C^{2}\left(\bar{\Omega}, \mathbb{R}^{n}\right) ; S_{1}^{\prime}(u)=0\right\} .
$$


Then it follows again that $C_{S_{1}^{\prime}}^{2}(\bar{\Omega})^{n}$ is dense in $F_{q, S_{1}^{\prime}}^{s}$, provided $1<q<\infty$ and $s \in[0,2] \backslash \Sigma_{1, q}$. Owing to (11) and the definition of $F_{q, S_{1}^{\prime}}^{s}$, we obtain that

$$
C_{S_{1}^{\prime}}^{2}(\bar{\Omega})^{n} \hookrightarrow F_{p_{0}^{\prime}, S_{1}^{\prime}}^{-s_{0}} \hookrightarrow F_{p_{1}^{\prime}, S_{1}^{\prime}}^{-s_{1}}
$$

We set

$$
C_{\mathcal{B}_{1}^{\prime}}^{2}(\bar{\Omega})^{n}:=\Lambda^{-1} C_{S_{1}^{\prime}}^{2}(\bar{\Omega})^{n}
$$

where $\Lambda$ is the isomorphism of Lemma 2.4. Hence, the density of $\left.C_{S_{1}^{\prime}}^{2} \bar{\Omega}\right)^{n}$ in $F_{p, S_{1}^{\prime}}^{s}$, embedding (14) and equation (10) yield that

$$
F_{p_{0}^{\prime}, \mathcal{B}_{1}^{\prime}}^{-s_{0}} \stackrel{d}{\hookrightarrow} F_{p_{1}^{\prime}, \mathcal{B}_{1}^{\prime}}^{-s_{1}}
$$

On the other hand, formula (15) follows for $k=2$ immediately from (a), since $F_{p^{\prime}, \mathcal{B}_{2}^{\prime}}^{s}=F_{p^{\prime}, \mathcal{B}_{2}}^{s}$. Therefore, we obtain the first assertion by duality and reflexivity.

(c) For $k=2$ the second statement follows by an obvious modification of the proof of Theorem 3.10 in [3].

In the sequel, we denote by $\left[\left(\boldsymbol{E}_{\alpha, k, p}, \boldsymbol{A}_{\alpha, k, p}\right) ; \alpha \in \mathbb{R}\right]$ the interpolation-extrapolation scale generated by $\left(L_{p, \mathcal{C}_{k}}, \boldsymbol{A}_{k, p}\right)$ and the interpolation functor $(\cdot, \cdot)_{\theta}, 0<\theta<1$, as chosen in (8). (We refer to chapter $\mathrm{V}$ in [1] for the definitions and fundamental properties of interpolation-extrapolation scales). We also use the abbreviations $\boldsymbol{E}_{\alpha}:=\boldsymbol{E}_{\alpha, k}:=\boldsymbol{E}_{\alpha, k, p}$ and $\boldsymbol{A}_{\alpha}:=\boldsymbol{A}_{\alpha, k}:=\boldsymbol{A}_{\alpha, k, p}$, if the skipped indices are obvious from the context.

Corollary 2.6. Suppose that $-2 \leq 2 \alpha \leq 2$ and that $2 \alpha \notin \Sigma_{k, p}$. Then

$$
\boldsymbol{E}_{\alpha, k, p} \doteq F_{p, \mathcal{C}_{k}}^{2 \alpha} .
$$

Proof. The assertion follows from Lemma 2.4 and the results in [1, Section V.2].

We want to recall a few important facts on interpolation-extrapolation scales, which will be used later on, and which show the main advantages of applying the theory of semigroups and interpolation-extrapolation scales, cf. [1, Section V.2].

Suppose that $A$ is the negative infinitesimal generator of an analytic semigroup $\left\{e^{-t A} ; t \geq 0\right\}$ on the reflexive Banach space $E$, and that $\left[\left(E_{\alpha}, A_{\alpha}\right) ; \alpha \in \mathbb{R}\right]$ is the interpolation-extrapolation scale generated by $(E, A)$ and the interpolation functor $(\cdot, \cdot)_{\theta}, 0<\theta<1$.

Then $-A_{\alpha}, \alpha \in \mathbb{R}$, generates an analytic semigroup $\left\{e^{-t A_{\alpha}} ; t \geq 0\right\}$ on $E_{\alpha}$, and 
the diagrams
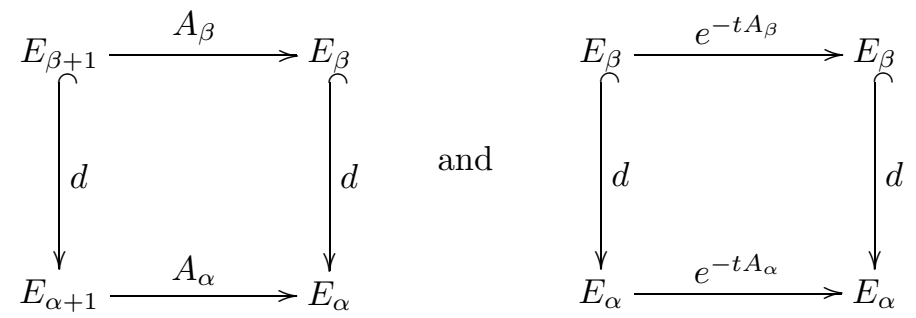

are commutative for $-\infty<\alpha<\beta<\infty$ and $t \geq 0$.

These properties are the abstract basis for weak formulations of quite general initial-boundary value problems and "bootstrapping" arguments leading to rather precise regularity results.

Drawing the consequences to the concrete situation under consideration, we obtain that

$$
\boldsymbol{A}_{\alpha} \in \mathcal{H}\left(\boldsymbol{E}_{\alpha+1}, \boldsymbol{E}_{\alpha}\right), \quad \alpha \geq-1
$$

and, thanks to Corollary 2.6, that

$$
\boldsymbol{A}_{\alpha, k, p} \in \mathcal{H}\left(F_{p, \mathcal{C}_{k}}^{2 \alpha+2}, F_{p, \mathcal{C}_{k}}^{2 \alpha}\right), \quad \alpha \geq-1
$$

\section{Existence and uniqueness}

The following proposition investigates the continuity properties of the nonlinear convection term $B_{k}:=P_{k} b$. Knowing the mapping properties of $B_{k}$ is crucial for the study of the Navier-Stokes equations.

Proposition 3.1. Let $0 \leq s \leq 2$ such that

$$
s>\max \left\{-1+\frac{n}{p},-\frac{n-1}{2}+\frac{n}{p}, \frac{2 n}{(n+1) p}\right\}=: s_{\min }(n, p) .
$$

Then for every $0<\delta \leq \min \{s+1-n / p, 1\}$

$$
B_{k}=P_{k} b \in \mathcal{L}^{2}\left(H_{p, \mathcal{C}_{k}}^{s}, H_{p, \mathcal{C}_{k}}^{s-2+\delta}\right),
$$

provided that $s$ and $s-2+\delta$ are not singular in the sense of Remark 2.3 (a) and that $\delta<1$ if $s=n / p$.

Proof. (a) Let us first suppose that $s<n / p$. Then thanks to inequalities (16) and $\delta \leq \min \{s+1-n / p, 1\}$ there exists $\rho \in(1, \infty)$ such that

$$
2 / p-s / n-(1-\delta) / n \leq 1 / \rho \leq 2 / p-s / n
$$


and

$$
1 / \rho<2(s-n / p)+n / \rho .
$$

Therefore setting $\tau:=2(s-n / p)+n / \rho$ and applying [18, Theorems 3.4.1 and 3.5.1] we obtain that the pointwise multiplication, and for that reason also the tensor product $\otimes$, is a continuous map from $H_{p}^{s} \times H_{p}^{s}$ into $H_{\rho}^{\tau}$. Hence, we infer due to $-1+1 / \rho<\tau-1 \leq 1$ and Remark 2.3(b) and (c) that

$$
B_{k} \in \mathcal{L}^{2}\left(H_{p, \mathcal{C}_{k}}^{s}, H_{\rho, \mathcal{C}_{k}}^{\tau-1}\right) .
$$

Now the assertion follows from Theorem 2.5 using inequality (17).

(b) Suppose that $s>n / p$. With the aid of $\delta \leq \min \{s+1-n / p, 1\}$ we deduce that there exists $\rho \in(1, \infty)$ such that

$$
1 / p-(1-\delta) / n \leq 1 / \rho \leq 1 / p
$$

and

$$
1 / \rho<(s-n / p)+n / \rho .
$$

Thus defining $\tau:=(s-n / p)+n / \rho$, invoking the results on pointwise multiplication of Johnsen, and Remark 2.3 once more, we see that (18) is true also in this case. Moreover, owing to (19) and Theorem 2.5 we derive that $H_{\rho, \mathcal{C}_{k}}^{\tau-1} \hookrightarrow H_{p, \mathcal{C}_{k}}^{s-2+\delta}$, so that

$$
B_{k} \in \mathcal{L}^{2}\left(H_{p, \mathcal{C}_{k}}^{s}, H_{p, \mathcal{C}_{k}}^{s-2+\delta}\right) .
$$

(c) Finally let us assume that $s=n / p$ and that $0<\delta<1$. Obviously, there exists $\bar{s} \in[0,2]$ such that

$$
s>\bar{s}>\max \left\{s-(1-\delta) / 3, s_{\min }(n, p)\right\} .
$$

We set $\bar{\delta}:=\bar{s}+1-n / p=\min \{\bar{s}+1-n / p, 1\}$. Then $B_{k}$ is a continuous bilinear map from $H_{p, \mathcal{C}_{k}}^{\bar{s}} \times H_{p, \mathcal{C}_{k}}^{\bar{s}}$ into $H_{p, \mathcal{C}_{k}}^{\bar{s}-2+\bar{\delta}}$, according to what we have already shown in (a). Now the assertion follows again from Theorem 2.5 using that $s>\bar{s}$ and that $\bar{s}-2+\bar{\delta}>s-2+\delta$.

For the remainder of this paper, we fix a real number $\mathrm{T}>0$ and set $\mathrm{J}:=[0, \mathrm{~T}]$. We also put $J_{T}:=[0, T]$ for any $T>0$.

Suppose that $E$ is a Banach space. We employ the following convention: For $T \in \mathbf{J}:=J \backslash\{0\}$ let $X_{T}$ and $Y_{T}$ be Banach spaces of $E$-valued distributions on $J_{T}$ being continuously embedded in $L_{1}\left(J_{T}, E\right)$. Also suppose that $M \in \mathcal{L}\left(X_{\mathrm{T}}, Y_{\mathrm{T}}\right)$. We write

$$
M \in \mathcal{L}\left(X_{T}, Y_{T}\right) \quad \text { J-uniformly }
$$

if $M\left(X_{T}\right) \subset Y_{T}$ and $\|M\|_{\mathcal{L}\left(X_{T}, Y_{T}\right)} \leq c$ for each $T \in \mathbf{j}$.

Corollary 3.2. Let $s_{\min }(n, p)<s \leq 2$ and $0<\delta \leq \min \{s+1-n / p, 1\}$ such that none of the values $s$ and $s-2+\delta$ is singular and $\delta<1$ if $s=n / p$. Then:

$$
B_{k} \in \mathcal{L}^{2}\left(L_{2 r}\left(J_{T}, H_{p, \mathcal{C}_{k}}^{s}\right), L_{r}\left(J_{T}, H_{p, \mathcal{C}_{k}}^{s-2+\delta}\right)\right) \quad \text { J-uniformly. }
$$


Proof. Of course, the mapping has to be understood in the sense of a Nemyt'skii (superposition) operator, that is,

$$
B_{k}(u, v)(t):=B_{k}(u(t), v(t)), \quad t \in J_{T}
$$

The assertion is now a consequence of Proposition 3.1 and Hölder's inequality.

Let $\left(E_{0}, E_{1}\right)$ be a densely injected Banach couple. Then $E_{[\theta]}:=\left[E_{0}, E_{1}\right]_{\theta}$ is the complex, $E_{\theta, \infty}^{0}:=\left(E_{0}, E_{1}\right)_{\theta, \infty}^{0}$ the continuous, and $E_{\theta, q}:=\left(E_{0}, E_{1}\right)_{\theta, q}$ the real interpolation space of $\left(E_{0}, E_{1}\right)$ of exponent $\theta \in(0,1)$ (and parameter $q \in[1, \infty]$ ). Recall that

$$
E_{1} \stackrel{d}{\hookrightarrow} E_{\theta, 1} \stackrel{d}{\hookrightarrow} E_{\theta, q} \stackrel{d}{\hookrightarrow} E_{\theta, r} \stackrel{d}{\hookrightarrow} E_{\theta, \infty}^{0} \hookrightarrow E_{\theta, \infty} \stackrel{d}{\hookrightarrow} E_{\vartheta, 1} \stackrel{d}{\hookrightarrow} E_{0}
$$

for $1<q<r<\infty$ and $0<\vartheta<\theta<1$. Moreover,

$$
E_{\theta, 1} \stackrel{d}{\hookrightarrow} E_{[\theta]} \stackrel{d}{\hookrightarrow} E_{\theta, \infty}^{0}, \quad 0<\theta<1 .
$$

For convenience, we also set $E_{[j]}:=E_{j, q}:=E_{j}$ for $j=0,1$ and $1 \leq q \leq \infty$.

For $1<r<\infty$ and a subinterval $I$ of $\mathrm{J}$ containing 0 such that $I \neq\{0\}$ we define

$$
\mathbb{W}_{r}^{1}\left(I,\left(E_{1}, E_{0}\right)\right):=L_{r}\left(I, E_{1}\right) \cap W_{r}^{1}\left(I, E_{0}\right) .
$$

Observe that

$$
\mathbb{W}_{r}^{1}\left(I,\left(E_{1}, E_{0}\right)\right) \doteq\left(\left\{u \in L_{r}\left(I, E_{1}\right) ; \partial u \in L_{r}\left(I, E_{0}\right)\right\},\|\cdot\|_{\mathbb{W}_{r}^{1}}\right)
$$

where $\partial$ is the distributional derivative, and

$$
\|u\|_{\mathbb{W}_{r}^{1}}:=\|u\|_{L_{r}\left(I, E_{1}\right)}+\|\partial u\|_{L_{r}\left(I, E_{0}\right)} .
$$

Similarly, we write $\mathbb{W}_{r, \text { loc }}^{1}\left(I,\left(E_{1}, E_{0}\right)\right):=L_{r, \text { loc }}\left(I, E_{1}\right) \cap W_{r, \text { loc }}^{1}\left(I, E_{0}\right)$.

We recall from [1, Section III.1.2] that $u \in W_{r}^{1}\left(I, E_{0}\right)$ if and only if $u$ is locally absolutely continuous and $u$ as well as the pointwise derivative $\dot{u}$ belong to $L_{r}\left(I, E_{0}\right)$. In this case $\partial u=\dot{u}$.

Suppose that $0<\theta<1$ and that $1<r<q<\infty$, then it is known from (the proof of) [2, Theorem 5.2] and [1, Theorem III.4.10.2] that the following important embeddings are valid

$$
\mathbb{W}_{r}^{1}\left(I,\left(E_{1}, E_{0}\right)\right) \hookrightarrow\left\{\begin{array}{l}
L_{q}\left(I, E_{\theta, 1}\right), \\
B U C\left(I, E_{1 / r^{\prime}, r}\right) .
\end{array} \quad \theta<1 / r^{\prime}+1 / q,\right.
$$

Suppose that $g \in L_{r}\left(\mathrm{~J}, E_{0}\right)$ and

$$
Q \in \mathcal{L}^{2}\left(\mathbb{W}_{r}^{1}\left(J_{T},\left(E_{1}, E_{0}\right)\right), L_{r}\left(J_{T}, E_{0}\right)\right) \quad \text { J-uniformly }
$$

are given. Then we consider the semilinear evolution equation

$$
\dot{u}+A u=g+Q(u, u) \text { in } \dot{\mathrm{j}} .
$$


By a $\mathbb{W}_{r}^{1}$-solution (or more precisely, a $\mathbb{W}_{r}^{1}\left(E_{1}, E_{0}\right)$-solution) on $I$ of (23), we mean an element $u \in \mathbb{W}_{r, \text { loc }}^{1}\left(I,\left(E_{1}, E_{0}\right)\right)$ satisfying (23) on $I$. It is maximal if there does not exist another such solution being a proper extension of it. If $\operatorname{dom}(u)=\mathrm{J}$ then $u$ is called a global solution. If $u$ is a $\mathbb{W}_{r}^{1}$-solution of (23) on $I$ then $u \in C\left(I, E_{1 / r^{\prime}, r}\right)$, thanks to (22). Thus, if $u^{0} \in E_{1 / r^{\prime}, r}$, then by a $\mathbb{W}_{r}^{1}$-solution of the initial value problem

$$
\left.\begin{array}{rl}
\dot{u}+A u & =g+Q(u, u), \quad \text { in } \mathbf{j}, \\
u(0) & =u^{0} .
\end{array}\right\}
$$

we mean a $\mathbb{W}_{r}^{1}$-solution $u$ of $(23)$ on $I$ such that $u(0)=u^{0}$.

We denote by $\{U(t) ; t \geq 0\}$ the semigroup on $E_{0}$ generated by $-A$. Given any $\varphi \in L_{1}\left(\dot{I}, E_{0}\right)$ we define

$$
U \star \varphi(t):=\int_{0}^{t} U(t-\tau) \varphi(\tau) d \tau, \quad t \in \dot{I},
$$

whenever these integrals exist. Moreover, we write $U x:=U(\cdot) x$ for $x \in E_{0}$. Then the maps $U:=(x \mapsto U x)$ and $U \star:=(\varphi \mapsto U \star \varphi)$ enjoy the following properties.

Lemma 3.3. Suppose that $1<r<\infty$ and that $0<\gamma<1$. Then

$$
U \star \in \mathcal{L}\left(L_{r}\left(J_{T}, E_{\gamma, \infty}\right), \mathbb{W}_{r}^{1}\left(J_{T},\left(E_{1}, E_{0}\right)\right)\right) \quad \text { J-uniformly. }
$$

Furthermore, for $0 \leq \alpha<1 / r$ we have that

$$
U \in \mathcal{L}\left(E_{1-\alpha, \infty}, \mathbb{W}_{r}^{1}\left(J_{T},\left(E_{1}, E_{0}\right)\right)\right) \quad \text { J-uniformly. }
$$

Proof. The assertions follow from [7, Lemma 4].

After these preparations we can formulate one of the main result of this paper, concerning the existence and uniqueness of maximal $\mathbb{W}_{r}^{1}$-solutions of the Cauchy problem

$$
\left.\begin{array}{rl}
\dot{u}+A_{k} u & =P_{k} f-B_{k}(u), \quad \text { in } \dot{j}, \\
u(0) & =u^{0} .
\end{array}\right\}
$$

Here, we have used the abbreviation $B_{k}(u):=B_{k}(u, u)$.

Theorem 3.4. Suppose that $1<p, r<\infty$ and that $0 \leq s \leq 2$ with

$$
\frac{2}{r}+\frac{n}{p}<3
$$

and

$$
\max \left\{-1+\frac{n}{p}+\frac{1}{r},-\frac{n-1}{2}+\frac{n}{p}, \frac{2 n}{(n+1) p}\right\}<s-\frac{1}{r} .
$$

Furthermore, let $0 \leq \alpha<1 / r$ and $\gamma>0$ with

$$
\left(u^{0}, P_{k} f\right) \in F_{p, \mathcal{C}_{k}}^{s-2 \alpha} \times L_{r}\left(\mathrm{~J}, F_{p, \mathcal{C}_{k}}^{s-2+2 \gamma}\right),
$$


and assume that none of the numbers $s, s-2, s-2 \alpha$, and $s-2+2 \gamma$ is singular. Then:

(i) Problem $(C P)_{k}$ possesses a unique maximal $\mathbb{W}_{r}^{1}\left(H_{p, \mathcal{C}_{k}}^{s}, H_{p, \mathcal{C}_{k}}^{s-2}\right)$-solution

$$
u=u\left(\cdot, u^{0}, f\right) \in \mathbb{W}_{r, l o c}^{1}\left(J^{+},\left(H_{p, \mathcal{C}_{k}}^{s}, H_{p, \mathcal{C}_{k}}^{s-2}\right)\right),
$$

and the maximal interval of existence, $J^{+}:=\operatorname{dom}(u)$, is open in $\mathrm{J}$.

(ii) For each $T \in \mathrm{j}$ there exists $R>0$ such that $J^{+} \supset J_{T}$ whenever

$$
\left\|u^{0}\right\|_{F_{p, \mathcal{C}_{k}}^{s-2 \alpha}}+\left\|P_{k} f\right\|_{L_{r}\left(J, F_{p, \mathcal{C}_{k}}^{s-2+2 \gamma}\right)} \leq R
$$

Proof. We observe that, given $1<p, r<\infty$ with $2 / r+n / p<3$, there exists $s \in[0,2] \backslash \Sigma_{k, p}$ satisfying inequality (24). Thus the statement of the theorem is never empty.

We set $\left(E_{0}, E_{1}\right):=\left(H_{p, \mathcal{C}_{k}}^{s-2}, H_{p, \mathcal{C}_{k}}^{s}\right), A:=\boldsymbol{A}_{k, s / 2-1} \in \mathcal{H}\left(E_{1}, E_{0}\right)$, and denote by $\{U(t) ; t \geq 0\}$ the semigroup on $E_{0}$ generated by $-A$.

Suppose that $0<\theta<1$ with $s-2+2 \theta \notin \Sigma_{k, p}$. It follows from the reiteration theorems for the real and complex interpolation functors that

$$
E_{\theta, 1}=\left(E_{0}, E_{1}\right)_{\theta, 1} \stackrel{d}{\hookrightarrow} F_{p, \mathcal{C}_{k}}^{s-2+2 \theta} \stackrel{d}{\hookrightarrow}\left(E_{0}, E_{1}\right)_{\theta, \infty}^{0}=E_{\theta, \infty}^{0},
$$

see for example [3, Lemma 1.1].

(a) Since inequality (24) is strict there exist $\varepsilon \in\left(0,1 / r^{\prime}\right)$ and $\delta>1 / r$ such that

$$
\max \left\{-1+\frac{n}{p}+\frac{1}{r}+\varepsilon,-\frac{n-1}{2}+\frac{n}{p}, \frac{2 n}{(n+1) p}\right\}<s-\frac{1}{r}-\varepsilon .
$$

as well as

$$
\delta<\min \left\{s-\frac{1}{r}+1-\frac{n}{p}-2 \varepsilon, 1-\varepsilon\right\},
$$

and such that neither $s-1 / r-\varepsilon$ nor $s-1 / r-2+\delta$ are singular. Then, in particular, we infer from (26) and Corollary 3.2 that

$$
B_{k} \in \mathcal{L}^{2}\left(L_{2 r}\left(J_{T}, H_{p, \mathcal{C}_{k}}^{s-1 / r-\varepsilon}\right), L_{r}\left(J_{T}, H_{p, \mathcal{C}_{k}}^{s-2+(\delta-1 / r)}\right)\right) \quad \text { J-uniformly. }
$$

Putting $\eta:=(2-1 / r-\varepsilon) / 2$ and $\xi:=\min \{\gamma,(\delta-1 / r) / 2\}$ implies by (25) that

$$
E_{\eta, 1} \stackrel{d}{\hookrightarrow} H_{p, \mathcal{C}_{k}}^{s-1 / r-\varepsilon}, \quad \text { and } \quad H_{p, \mathcal{C}_{k}}^{s-2+(\delta-1 / r)} \stackrel{d}{\hookrightarrow} E_{\xi, \infty}^{0} .
$$

Therefore, it follows from Lemma 3.3, with the aid of the formulas (22) and (25), that $Q:=U \star B_{k} \in \mathcal{L}^{2}\left(L_{2 r}\left(J_{T}, E_{\eta, 1}\right), L_{2 r}\left(J_{T}, E_{\eta, 1}\right)\right)$. However, a more careful analysis of the mapping properties of $U \star$ reveals that the norm of $Q$ is, in fact, independent of $T \in \dot{\mathrm{J}}$, that is,

$$
Q=U \star B_{k} \in \mathcal{L}^{2}\left(L_{2 r}\left(J_{T}, E_{\eta, 1}\right), L_{2 r}\left(J_{T}, E_{\eta, 1}\right)\right) \quad \text { J-uniformly, }
$$


see Lemmas 1 and 3 in [7]. Now let us consider $a:=U u^{0}+U \star P_{k} f$. We derive from (25) and (20) that

$$
\left(u^{0}, P_{k} f\right) \in E_{1-\alpha, \infty} \times L_{r}\left(J_{T}, E_{\xi, \infty}\right) .
$$

Similarly as above, we infer from [7, Lemmas 1 and 3] that $a \in L_{2 r}\left(J_{T}, E_{\eta, 1}\right)$ and that there exists $\bar{\varepsilon}>0$ with

$$
\|a\|_{L_{2 r}\left(J_{T}, E_{\eta, 1}\right)} \leq c T^{\bar{\varepsilon}}\left(\left\|u^{0}\right\|_{F_{p, \mathcal{C}_{k}}^{s-2 \alpha}}+\left\|P_{k} f\right\|_{L_{r}\left(J_{T}, F_{p, \mathcal{C}_{k}}^{s-2+2 \gamma}\right)}\right), \quad T \in \mathbf{j} .
$$

Owing to $\|a\|_{L_{2 r}\left(J_{T}, E_{\eta, 1}\right)} \rightarrow 0$ as $T \rightarrow 0$, we deduce by a fixed point argument, [8, Lemma 2.1], that there exists $T_{0} \in \mathrm{j}$ and a unique $u_{0} \in L_{2 r}\left(J_{T_{0}}, E_{\eta, 1}\right)$ such that

$$
u_{0}=a+Q\left(u_{0}, u_{0}\right)
$$

and

$$
\left\|u_{0}-a\right\|_{L_{2 r}\left(J_{T_{0}}, E_{\eta, 1}\right)}<\frac{2-\sqrt{3}}{4\|Q\|} .
$$

Due to the above construction, the solution $u_{0}$ belongs to $L_{2 r}\left(J_{T_{0}}, E_{\eta, 1}\right)$. However, Lemma 3.3 guarantees, in view of (28), that $u_{0}$ possesses more regularity, namely

$$
u_{0}=U u^{0}+U \star\left(P_{k} f-B_{k}\left(u_{0}\right)\right) \in \mathbb{W}_{r}^{1}\left(J_{T_{0}},\left(E_{1}, E_{0}\right)\right) .
$$

Setting $g:=P_{k} f-B_{k}\left(u_{0}\right)$ we infer that $u_{0}$ is a mild solution of the linear initial value problem

$$
\dot{u}+A u=g, \quad t \in \dot{J}_{T_{0}}, \quad u(0)=u^{0} .
$$

If $\left(u^{0}, g\right) \in E_{1} \times C^{1}\left(J_{T_{0}}, E_{1}\right)$ then, it follows from [1, Theorem II.1.2.1], that $u_{0}$ is a strict classical solution of $(30)$ on $J_{T_{0}}$, that is, $u_{0} \in C^{1}\left(J_{T_{0}}, E_{0}\right) \cap C\left(J_{T_{0}}, E_{1}\right)$ and

$$
\partial u_{0}+A u_{0}=g, \quad u_{0}(0)=u^{0} .
$$

We claim that, due to the density of $E_{1} \times C^{1}\left(J_{T_{0}}, E_{1}\right)$ in $E_{1-\alpha, \infty}^{0} \times L_{r}\left(J_{T_{0}}, E_{\xi, \infty}^{0}\right)$, this implies that $u_{0}$ is a $\mathbb{W}_{r}^{1}$-solution of $(C P)_{k}$. Indeed, there exists a sequence $\left(u_{j}^{0}, g_{j}\right)$ in $E_{1} \times C^{1}\left(J_{T_{0}}, E_{1}\right)$ such that

$$
\left(u_{j}^{0}, g_{j}\right) \rightarrow\left(u^{0}, g\right) \text { in } E_{1-\alpha, \infty}^{0} \times L_{r}\left(J_{T_{0}}, E_{\xi, \infty}^{0}\right) .
$$

By Lemma 3.3 we see that $v_{j}:=U u_{j}^{0}+U \star g_{j}$ converges in $\mathbb{W}_{r}^{1}\left(J_{T_{0}},\left(E_{1}, E_{0}\right)\right)$ towards $u_{0}$ and consequently

$$
g_{j}=\partial v_{j}+A v_{j} \rightarrow \partial u_{0}+A u_{0} \quad \text { in } L_{r}\left(J_{T_{0}}, E_{0}\right) .
$$

Hence, we deduce from (31) that $\dot{u}_{0}+A u_{0}=\partial u_{0}+A u_{0}=g$ so that $u_{0}$ is in fact a $\mathbb{W}_{r}^{1}$-solution of $(C P)_{k}$ on $J_{T_{0}}$.

(b) Next we show that the solution $u_{0}$ can be extended to some Interval $J_{T_{1}}$ with $T_{1}>T_{0}$. Thanks to (22) we know that $u_{0} \in B U C\left(J_{T_{0}}, E_{1 / r^{\prime}, r}\right)$, and in particular $u^{1}:=u_{0}\left(T_{0}\right) \in E_{1 / r^{\prime}, r}$. Unfortunately, this (spatial) regularity is not 
sufficient to apply the method in (a) of constructing a $\mathbb{W}_{r}^{1}\left(E_{1}, E_{0}\right)$-solution with initial value $u^{1}$. However, since the inequalities (24), $\alpha<1 / r$, and $\gamma>0$ are strict, we can increase the regularity of $u^{0}$ slightly: We choose $\mu>0$ such that $\mu<\min \{1 / r-\alpha, \eta\}$ and set

$$
\tilde{\alpha}:=\alpha+\mu, \quad \tilde{\eta}:=\eta-\mu, \quad \tilde{s}:=s+2 \mu .
$$

We also put $\left(\tilde{E}_{0}, \tilde{E}_{1}\right):=\left(H_{p, \mathcal{C}_{k}}^{\tilde{s}-2}, H_{p, \mathcal{C}_{k}}^{\tilde{s}}\right), \tilde{A}:=\boldsymbol{A}_{k, \tilde{s} / 2-1}$ and denote by $\{\tilde{U}(t) ; t \geq 0\}$ the semigroup on $\tilde{E}_{0}$ generated by $-\tilde{A}$, so that $\tilde{U}(t) \subset U(t)$ for $t \geq 0$. Now, invoking Lemma 3.3 once more, we see that

$$
u_{0}=\tilde{U} u^{0}+\tilde{U} \star\left(P_{k} f-B_{k}\left(u_{0}\right)\right) \in \mathbb{W}_{r}^{1}\left(J_{T_{0}},\left(\tilde{E}_{1}, \tilde{E}_{0}\right)\right) .
$$

We deduce from (20) and (25) that

$$
u^{1}=u_{0}\left(T_{0}\right) \in\left(\tilde{E}_{0}, \tilde{E}_{1}\right)_{1 / r^{\prime}, r} \hookrightarrow\left(E_{1}, E_{0}\right)_{1 / r^{\prime}+\mu / 2, \infty} .
$$

Thus, as in step (a), we infer that there exists $T_{1}>T_{0}$ and a unique $\mathbb{W}_{r}^{1}$-solution $v^{1} \in \mathbb{W}_{r}^{1}\left(J_{T_{1}-T_{0}},\left(E_{1}, E_{0}\right)\right)$ on $J_{T_{1}-T_{0}}$ of

$$
\left.\begin{array}{rl}
\dot{v}+A v & =P_{k} f\left(\cdot+T_{0}\right)-B_{k}(v), \text { in } \mathbf{j}, \\
v(0) & =u^{1}
\end{array}\right\}
$$

satisfying

$$
\left\|a_{1}\right\|_{L_{2 r}\left(J_{T_{1}-T_{0}}, E_{\eta, 1}\right)}<(2-\sqrt{3}) /(4\|Q\|), \text { where } a_{1}:=U u^{1}+U \star P_{k} f\left(\cdot+T_{0}\right) .
$$

Now setting

$$
u_{1}(t):= \begin{cases}u_{0}(t), & 0 \leq t \leq T_{0}, \\ v_{1}\left(t-T_{0}\right), & T_{0}<t \leq T_{1},\end{cases}
$$

yields that $u_{1}$ is a $\mathbb{W}_{r}^{1}\left(E_{1}, E_{0}\right)$-solution of $(C P)_{k}$ on $J_{T_{1}}$.

By iterating this argument we arrive at a maximal extension $u:=u\left(\cdot, u^{0}, f\right)$ of $u_{0}$ defined on $J^{+}$such that $u$ is a $\mathbb{W}_{r}^{1}\left(E_{1}, E_{0}\right)$-solution of $(C P)_{k}$ on $J^{+}$. Moreover, if $J^{+} \neq \mathrm{J}$ then the above arguments imply that $J^{+}$is open (in $\left.\mathbb{R}^{+}\right)$.

(c) Suppose that $v$ is another $\mathbb{W}_{r}^{1}\left(E_{1}, E_{0}\right)$-solution of $(C P)_{k}$ on some interval $I \subset \mathrm{J}$ such that $u \not \supset v$. Then it is known, e.g. Proposition III.1.3.1 in [1], that $v$ is a mild solution of $(C P)_{k}$, that is, $v=U u^{0}+U \star\left(P_{k} f-B_{k}(v)\right)$. Furthermore,

$$
T^{\prime}:=\max \left\{t \in I \cap J^{+} ; u(t)=v(t) \text { in } E_{1 / r^{\prime}, r}\right\}
$$

is well-defined and $I^{\prime}:=\left(I-T^{\prime}\right) \cap \mathbb{R}^{+}$is a nontrivial subinterval of $\mathbb{R}^{+}$containing 0 . We set $x:=u\left(T^{\prime}\right)$ and $h(t):=P_{k}\left(t+T^{\prime}\right)$ for $t \in I^{\prime}$. Then the initial value problem

$$
\left.\begin{array}{rl}
\dot{w}+A w & =h-B_{k}(w), \quad \text { in } \dot{I}^{\prime}, \\
w(0) & =x,
\end{array}\right\}
$$

has two distinct $\mathbb{W}_{r}^{1}\left(E_{1}, E_{0}\right)$-solutions $w_{1}:=u\left(\cdot+T^{\prime}\right)$ and $w_{2}:=v\left(\cdot+T^{\prime}\right)$ on $I^{\prime}$. We put $a^{\prime}:=U x+U \star h$ and observe, by formula (27) and Lebesgue's theorem, that

$$
\left\|w_{j}-a^{\prime}\right\|_{L_{2 r}\left(J_{T}, E_{\eta, 1}\right)} \rightarrow 0, \quad \text { as } T \rightarrow 0, \quad \text { for } j=1,2 .
$$


Thus there exists $T_{0}^{\prime} \in \dot{I}^{\prime}$ such that $\left\|w_{j}-a^{\prime}\right\|_{L_{2 r}\left(J_{T_{0}^{\prime}}, E_{\eta, 1}\right)}<(2-\sqrt{3}) /(4\|Q\|)$. Now the uniqueness assertion in step (a) implies that $w_{1}=w_{2}$ in $B U C\left(J_{T_{0}^{\prime}}, E_{1 / r^{\prime}, r}\right)$ which contradicts the definition of $T^{\prime}$. Hence, $u$ is the unique maximal $\mathbb{W}_{r}^{1}$-solution of $(C P)_{k}$.

(d) Lastly, owing to inequality (29) the second assertion is obvious.

Theorem 3.4 guarantees for each choice of $p, r$ and $s$ a unique maximal solution $u_{p, r, s}$. The following propositions show, however, that $u_{p, r, s}$ is independent of the parameters $p, r$ and $s$, provided the data are sufficiently regular. The proofs rely essentially on the smoothing properties of the semigroup generated by $-\boldsymbol{A}_{k}$, see for instance [7, Lemmas 1-4].

Proposition 3.5. Suppose that $1<r_{0}<r_{1}<\infty$ and $0 \leq s \leq 2$ such that

$$
\max \left\{-1+\frac{n}{p}+\frac{1}{r_{0}},-\frac{n-1}{2}+\frac{n}{p}, \frac{2 n}{(n+1) p}\right\}<s-\frac{1}{r_{0}} .
$$

Also suppose that $0 \leq \alpha<1 / r_{1}, \gamma>0$,

$$
\left(u^{0}, P_{k} f\right) \in F_{p, \mathcal{C}_{k}}^{s-2 \alpha} \times L_{r_{1}}\left(\mathrm{~J}, F_{p, \mathcal{C}_{k}}^{s-2+2 \gamma}\right),
$$

and that none of the values $s, s-2, s-2 \alpha$, and $s-2+2 \gamma$ is singular.

Let $u_{j}$ denote the maximal $\mathbb{W}_{r_{j}}^{1}$-solution of $(C P)_{k}$, according to Theorem 3.4. Then:

$$
u_{0}=u_{1} .
$$

Proof. It is obvious that $P_{k} f \in L_{r_{j}}\left(\mathrm{~J}, F_{p, \mathcal{C}_{k}}^{s-2+2 \gamma}\right)$ for $j=0,1$. Thus $u_{j}$ is welldefined. Denote the interval of existence of $u_{0}$ by $J_{0}^{+}$and fix some $T \in \dot{J}_{0}^{+}$. Then $u_{0}=U u^{0}+U \star\left(P_{k} f-B_{k}\left(u_{0}\right)\right) \in \mathbb{W}_{r_{0}}^{1}\left(J_{T},\left(H_{p, \mathcal{C}_{k}}^{s}, H_{p, \mathcal{C}_{k}}^{s-2}\right)\right)$, by [1, Prop. III.1.3.1]. Thanks to Lemma 3.3 we know that

$$
U u^{0}+U \star P_{k} f \in \mathbb{W}_{r_{1}}^{1}\left(J_{T},\left(H_{p, \mathcal{C}_{k}}^{s}, H_{p, \mathcal{C}_{k}}^{s-2}\right)\right) .
$$

Now let us show that $U \star\left(B_{k}\left(u_{0}\right)\right)$ belongs to this space as well. First, we cover the situation where the difference of $r_{0}$ and $r_{1}$ is rather small. To be precise, we assume that

$$
\frac{1}{r_{0}}-\frac{1}{r_{1}}<\min \left\{\left(s-\frac{1}{r_{0}}\right)-s_{\min }(n, p), 1-\frac{1}{r_{0}}, \frac{1}{2}\left(s-\left(-1+\frac{n}{p}+\frac{2}{r_{0}}\right)\right)\right\} .
$$

This implies together with inequality (32) that there exists $\tilde{s} \in \mathbb{R}$ such that

$$
\max \left\{s_{\min }(n, p), s-1, \frac{1}{2}\left(s-1+\frac{n}{p}\right)\right\}<\tilde{s}<s-\frac{2}{r_{0}}+\frac{1}{r_{1}} .
$$

In particular, we deduce from (22) and formula (25) that

$$
\mathbb{W}_{r_{0}}^{1}\left(J_{T},\left(H_{p, \mathcal{C}_{k}}^{s}, H_{p, \mathcal{C}_{k}}^{s-2}\right)\right) \hookrightarrow L_{2 r_{1}}\left(J_{T}, H_{p, \mathcal{C}_{k}}^{\tilde{s}}\right) .
$$


Then, by (34) and Corollary 3.2, we see that there exists some $\delta>0$ such that $B_{k}\left(u_{0}\right) \in L_{r_{1}}\left(J_{T}, H_{p, \mathcal{C}_{k}}^{s-2+\delta}\right)$. Hence, it follows from Lemma 3.3 that

$$
u_{0}=U u^{0}+U \star\left(P_{k} f-B_{k}\left(u_{0}\right)\right) \in \mathbb{W}_{r_{1}}^{1}\left(J_{T},\left(H_{p, \mathcal{C}_{k}}^{s}, H_{p, \mathcal{C}_{k}}^{s-2}\right)\right) .
$$

Consequently, $u_{0}$ is a $\mathbb{W}_{r_{1}}^{1}$-solution of problem $(C P)_{k}$ on $J_{0}^{+}$, and we obtain that $u_{0} \subset u_{1}$ by the maximality and uniqueness of $u_{1}$. Of course, the reverse inclusion is trivially satisfied since $\mathrm{J}$ is bounded.

Now suppose that (33) is not fulfilled. However, on account of (32) there exist $N \in \mathbb{N}$ and $\rho_{0}:=r_{0}<\rho_{1}<\ldots<\rho_{N-1}<\rho_{N}:=r_{1}$ such that (33) and the assumptions of this propositions are valid if we replace $\left(r_{0}, r_{1}\right)$ by $\left(\rho_{j}, \rho_{j+1}\right)$ for $j=0, \ldots, N-1$. Thus the assertion follows by finite induction.

Proposition 3.6. Let $1<r<\infty, 1<p_{0}<p_{1}<\infty$, and $0 \leq s \leq 2$ with

$$
\max \left\{-1+\frac{n}{p_{0}}+\frac{1}{r},-\frac{n-1}{2}+\frac{n}{p_{0}}, \frac{2 n}{(n+1) p_{0}}\right\}<s-\frac{1}{r} .
$$

Furthermore, suppose that $0 \leq \alpha<1 / r, \gamma>0$,

$$
\left(u^{0}, P_{k} f\right) \in F_{p_{1}, \mathcal{C}_{k}}^{s-2 \alpha} \times L_{r}\left(\mathrm{~J}, F_{p_{1}, \mathcal{C}_{k}}^{s-2+2 \gamma}\right),
$$

and that $s, s-2, s-2 \alpha$, and $s-2+2 \gamma$ are not singular.

If $u_{j}$ denotes the unique maximal $\mathbb{W}_{r}^{1}\left(J_{T},\left(H_{p_{j}, \mathcal{C}_{k}}^{s}, H_{p_{j}, \mathcal{C}_{k}}^{s-2}\right)\right)$-solution of $(C P)_{k}$, then

$$
u_{0}=u_{1} .
$$

Proof. We deduce from Theorems 2.5 and 3.4 that $u_{j}$ is well-defined for $j=0,1$. Similarly as in the proof of the preceding theorem, the assertion now follows from Lemma 3.3. We omit the details and refer instead to [22].

Proposition 3.7. Let $1<p, r<\infty$ and $0 \leq s_{0}<s_{1} \leq 2$ with

$$
\max \left\{-1+\frac{n}{p}+\frac{1}{r},-\frac{n-1}{2}+\frac{n}{p}, \frac{2 n}{(n+1) p}\right\}<s_{0}-\frac{1}{r} .
$$

Also assume that $0 \leq \alpha<1 / r, \gamma>0$,

$$
\left(u^{0}, P_{k} f\right) \in F_{p, \mathcal{C}_{k}}^{s_{0}-2 \alpha} \times L_{r}\left(\mathrm{~J}, F_{p, \mathcal{C}_{k}}^{s_{1}-2+2 \gamma}\right),
$$

and that none of the values $s_{j}, s_{j}-2, s_{0}-2 \alpha$, and $s_{1}-2+2 \gamma$ is singular. Then:

(i) The unique maximal $\mathbb{W}_{r}^{1}\left(H_{p, \mathcal{C}_{k}}^{s_{0}}, H_{p, \mathcal{C}_{k}}^{s_{0}-2}\right)$-solution $u=u\left(\cdot, u^{0}, f\right)$ of problem $(C P)_{k}$, according to Theorem 3.4, belongs to

$$
\mathbb{W}_{r, l o c}^{1}\left(\dot{J}^{+},\left(H_{p, \mathcal{C}_{k}}^{s_{1}}, H_{p, \mathcal{C}_{k}}^{s_{1}-2}\right)\right) \text {. }
$$

(ii) If $\alpha+\left(s_{1}-s_{0}\right) / 2<1 / r$ then $u \in \mathbb{W}_{r, l o c}^{1}\left(J^{+},\left(H_{p, \mathcal{C}_{k}}^{s_{1}}, H_{p, \mathcal{C}_{k}}^{s_{1}-2}\right)\right)$ and $u$ coincides with the unique maximal $\mathbb{W}_{r}^{1}\left(H_{p, \mathcal{C}_{k}}^{s_{1}}, H_{p, \mathcal{C}_{k}}^{s_{1}-2}\right)$-solution of $(C P)_{k}$. 
Proof. (a) By (25) and Theorem II.1.2.1 in [1] we know that

$$
U u^{0} \in C^{1}\left((0, T], H_{p, \mathcal{C}_{k}}^{s_{0}-2}\right) \cap C\left((0, T], H_{p, \mathcal{C}_{k}}^{s_{0}}\right), \quad T>0 .
$$

Let $0<T_{0}<T_{1}$ and set $u^{1}:=u\left(T_{0} / 2\right) \in H_{p, \mathcal{C}_{k}}^{s_{0}}$. Since $s_{1}-2<s_{0}<s_{1}$, we can apply (25) and [1, Thm.II.1.2.1] once more to obtain that

$$
U u^{1} \in C^{1}\left((0, T], H_{p, \mathcal{C}_{k}}^{s_{1}-2}\right) \cap C\left((0, T], H_{p, \mathcal{C}_{k}}^{s_{1}}\right), \quad T>0
$$

and therefore

$$
U u^{0} \in \mathbb{W}_{r}^{1}\left(\left[T_{0}, T_{1}\right],\left(H_{p, \mathcal{C}_{k}}^{s_{1}}, H_{p, \mathcal{C}_{k}}^{s_{1}-2}\right)\right),
$$

by using the fact that $U u^{1}(t)=U u^{0}\left(t+T_{0} / 2\right)$ for $t \geq 0$. Combining this with Lemma 3.3, we infer that

$$
a:=U u^{0}+U \star P_{k} f \in \mathbb{W}_{r}^{1}\left(\left[T_{0}, T_{1}\right],\left(H_{p, \mathcal{C}_{k}}^{s_{1}}, H_{p, \mathcal{C}_{k}}^{s_{1}-2}\right)\right),
$$

for each $T_{j} \in \mathbf{j}$ with $T_{0}<T_{1}$.

(b) Let $T_{0}, T_{1} \in \operatorname{dom}(u)$ with $0<T_{0}<T_{1}$ be arbitrary. In order to provide a comprehensive line of reasoning we define the following auxiliary functions

$$
\phi(s):=\max \left\{s_{\min }(n, p), s-1, \frac{1}{2}\left(s+1-\frac{n}{p}\right)\right\}, \quad 0 \leq s \leq 2,
$$

and

$$
\delta_{\max }(s):=\frac{1}{2}\left(\left(s-\frac{1}{r}\right)-\phi(s)\right), \quad 0 \leq s \leq 2 .
$$

Then it follows from inequality $(36)$ that $\phi\left(s_{0}\right)<s_{0}-1 / r$, or equivalently that $\delta_{\max }\left(s_{0}\right)>0$. Thus, there exist $\delta \in\left(0, \delta_{\max }\left(s_{0}\right)\right)$ and $N \in \mathbb{N}$ such that $s_{1}=s_{0}+N \delta$ and that $s_{0}+k \delta$ is not singular for every $k=1, \ldots, N-1$.

Now, we define $\tilde{s}:=\left(s_{0}-1 / r\right)-\delta / 2$ and observe that $\tilde{s}>\phi\left(s_{0}\right)+3 \delta / 2$. It follows, in particular, that $s_{0}-2<\tilde{s}<s_{0}$ and $s_{0}-2 / r>\tilde{s}-2 / 2 r$, so that

$$
u \in \mathbb{W}_{r}^{1}\left(J_{T_{1}},\left(H_{p, \mathcal{C}_{k}}^{s_{0}}, H_{p, \mathcal{C}_{k}}^{s_{0}-2}\right)\right) \hookrightarrow L_{2 r}\left(J_{T_{1}}, H_{p, \mathcal{C}_{k}}^{\tilde{s}}\right),
$$

by $(22)$ and (25). It is not difficult to verify that Corollary 3.2 and Lemma 3.3 imply, on account of $(22)$ and $\tilde{s}>\phi\left(s_{0}\right)+3 \delta / 2$, that

$$
U \star B_{k}(u) \in \mathbb{W}_{r}^{1}\left(J_{T_{1}},\left(H_{p, \mathcal{C}_{k}}^{s_{0}+\delta}, H_{p, \mathcal{C}_{k}}^{s_{0}-2+\delta}\right)\right) .
$$

Thanks to $u=a-U \star B_{k}(u)$ and (37) we conclude

$$
u \in \mathbb{W}_{r}^{1}\left(\left[T_{0}, T_{1}\right],\left(H_{p, \mathcal{C}_{k}}^{s_{0}+\delta}, H_{p, \mathcal{C}_{k}}^{s_{0}-2+\delta}\right)\right)
$$

which proves the first assertion if $N=1$. Otherwise, we repeat the above procedure $(N-1$ times $)$ to obtain that

$$
u \in \mathbb{W}_{r}^{1}\left(\left[T_{0}, T_{1}\right],\left(H_{p, \mathcal{C}_{k}}^{s_{0}+k \delta}, H_{p, \mathcal{C}_{k}}^{s_{0}-2+k \delta}\right)\right), \quad k=1, \ldots, N
$$


This is possible because inequality (36) remains true if we replace $s_{0}$ by $s_{0}+k \delta$, and because the map $\left(s \mapsto \delta_{\max }(s)\right)$ is non-decreasing on $\left[s_{0}, 2\right]$, so that

$$
\delta<\delta_{\max }\left(s_{0}+k \delta\right) \text { for every } k \in\{0, \ldots, N-1\} .
$$
that

(c) Suppose now that $\tilde{\alpha}:=\alpha+\left(s_{1}-s_{0}\right) / 2<1 / r$. Then we get by formula (25)

$$
F_{p, \mathcal{C}_{k}}^{s_{0}-2 \alpha} \hookrightarrow\left(H_{p, \mathcal{C}_{k}}^{s_{1}-2}, H_{p, \mathcal{C}_{k}}^{s_{1}}\right)_{1-\tilde{\alpha}, \infty}^{0} .
$$

Thus invoking Lemma 3.3 once more, yields

$$
a \in \mathbb{W}_{r}^{1}\left([0, T],\left(H_{p, \mathcal{C}_{k}}^{s_{1}}, H_{p, \mathcal{C}_{k}}^{s_{1}-2}\right)\right), \quad T>0 .
$$

The bootstrapping method used in (b) implies, therefore, that $u$ belongs to

$$
\mathbb{W}_{r, \text { loc }}^{1}\left(J^{+},\left(H_{p, \mathcal{C}_{k}}^{s_{1}}, H_{p, \mathcal{C}_{k}}^{s_{1}-2}\right)\right) \text {. }
$$

Hence, $u$ is a $\mathbb{W}_{r}^{1}\left(H_{p, \mathcal{C}_{k}}^{s_{1}}, H_{p, \mathcal{C}_{k}}^{s_{1}-2}\right)$-solution of problem $(C P)_{k}$ on $J^{+}$. Now the second assertion follows from the uniqueness and maximality of $u$.

Finally, we draw the consequences for the original Navier-Stokes equations

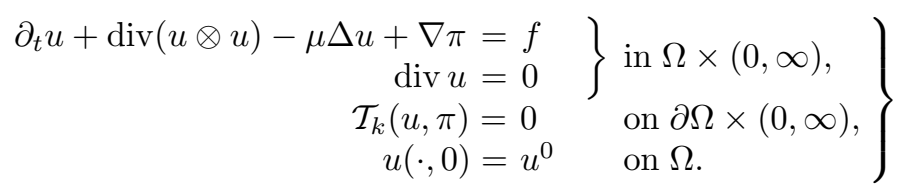

That is, we translate the results of Theorem 3.4 back to the system $(N S E)_{k}$.

Theorem 3.8. Let $1<p, r<\infty$ with $2 / r+n / p<3$ and

$$
\max \left\{-1+\frac{n}{p}+\frac{2}{r}, 1+\frac{1}{p}\right\}<s \leq 2 .
$$

Moreover, suppose that $0 \leq \alpha<1 / r$ and $\gamma>0$ with

$$
\left(u^{0}, P_{k} f\right) \in F_{p, \mathcal{C}_{k}}^{s-2 \alpha} \times L_{r}\left(\mathrm{~J}, F_{p, \mathcal{C}_{k}}^{s-2+2 \gamma}\right), \quad \operatorname{div}\left(u^{0}\right)=0,
$$

and none of the values $s, s-2, s-2 \alpha$, and $s-2+2 \gamma$ is singular.

Then problem $(N S E)_{k}$ possesses a unique maximal solution

$$
(u, \pi) \in \mathbb{W}_{r, l o c}^{1}\left(J^{+},\left(H_{p, \mathcal{C}_{k}}^{s}, H_{p, \mathcal{C}_{k}}^{s-2}\right)\right) \times L_{r, l o c}\left(J^{+}, H_{p}^{s-1}(\Omega)\right) .
$$

subject to the condition

$$
\int_{\Omega} \pi d x=0, \quad \text { for a.a. } t \in j^{+} \text {if } k=2 .
$$

Furthermore, the maximal interval of existence is open in $\mathbf{j}$; and $(u, \pi)$ is a global solution, that is, $\operatorname{dom}(u)=\mathrm{J}=[0, \mathrm{~T}]$, if the data are sufficiently small. 
Proof. (a) Let us suppose that $k=1$. Then, by definition, we have that

$$
\boldsymbol{A}_{0} v=-\mu \Delta v+2 \mu \nabla K_{D}(\gamma D(u) \nu)_{\nu}, \quad v \in \operatorname{dom}\left(\boldsymbol{A}_{0}\right)=H_{p, \mathcal{C}_{1}}^{2} .
$$

Since $\boldsymbol{A}_{s / 2-1} \in \mathcal{L}\left(H_{p, \mathcal{C}_{1}}^{s}, H_{p, \mathcal{C}_{1}}^{s-2}\right)$ is the uniquely determined continuous extension of $\boldsymbol{A}_{0} \in \mathcal{L}\left(H_{p, \mathcal{C}_{1}}^{2}, L_{p}\right)$ to $H_{p, \mathcal{C}_{1}}^{s}$ we deduce from (3) that the extrapolated operator $\boldsymbol{A}_{s / 2-1}$ also has the form

$$
\boldsymbol{A}_{s / 2-1} v=-\mu \Delta v+2 \mu \nabla K_{D}(\gamma D(v) \nu)_{\nu}, \quad v \in \operatorname{dom}\left(\boldsymbol{A}_{s / 2-1}\right)=H_{p, \mathcal{C}_{1}}^{s} .
$$

Simple calculations show that

$$
\max \left\{-1+\frac{n}{p}+\frac{2}{r}, 1+\frac{1}{p}\right\}>\max \left\{-\frac{n-1}{2}+\frac{n}{p}+\frac{1}{r}, \frac{2 n}{(n+1) p}+\frac{1}{r}\right\} .
$$

Therefore, Theorem 3.4 guarantees the existence of a unique maximal $\mathbb{W}_{r}^{1}$-solution

$$
u=u\left(\cdot, u^{0}, f\right) \in \mathbb{W}_{r, \text { loc }}^{1}\left(H_{p, \mathcal{C}_{1}}^{s}, H_{p, \mathcal{C}_{1}}^{s-2}\right)
$$

of the reduced Navier-Stokes equations $(C P)_{1}$. It follows from (39) that

$$
\partial_{t} u-\mu \Delta u+2 \mu \nabla K_{D}(\gamma D(u) \nu)_{\nu}=P_{1}(f-b(u)) \quad \text { in } L_{r, l o c}\left(J^{+}, H_{p, \mathcal{C}_{1}}^{s-2}\right) .
$$

Now setting $v:=\operatorname{div}(u) \in \mathbb{W}_{r, \text { loc }}^{1}\left(J^{+},\left(H_{p}^{s-1}(\Omega), H_{p}^{s-3}(\Omega)\right)\right)$ implies that $v$ is a $\mathbb{W}_{r}^{1}$-solution of the homogeneous heat equation with zero Dirichlet boundary conditions and zero initial function $v^{0}=0$. Indeed, applying the divergence operator to equation (40) and using that $\operatorname{div}\left(\partial_{t} u\right)=\partial_{t} \operatorname{div}(u)$ as well as $-\Delta K_{D}=0$ and $\operatorname{div} P_{k}=0$, entails that

$$
\partial_{t} v-\mu \Delta v=0
$$

Since $s>1+1 / p$ and $u \in L_{r, l o c}\left(J^{+}, H_{p, \mathcal{C}_{1}}^{s}\right)$ we infer that $\mathcal{B}_{1}(u)=0$ and therefore that $\gamma v=\gamma \operatorname{div}(u)=0$ and

$$
v(0)=\operatorname{div}(u)(0)=\operatorname{div}(u(0))=\operatorname{div}\left(u^{0}\right)=0 .
$$

Hence, we derive from the unique $\left(\mathbb{W}_{r^{-}}^{1}\right)$ solvability of the Dirichlet problem for the heat equation, cf. [1, Proposition III.1.3.1], that $v=0$. Hence, $u$ satisfies the incompressibility constraint $\operatorname{div}(u)=0$.

Defining the pressure $\pi$ by

$$
\pi:=2 \mu K_{D}(\gamma D(u) \nu)_{\nu}-R_{D} \operatorname{div}(f-b(u)) .
$$

we deduce from (3) and (4) that $\pi \in L_{r, l o c}\left(J^{+}, H_{p}^{s-1}(\Omega)\right)$. Moreover, we infer, by the definition of $\pi$ and the solution operator $K_{D}$, that $(\gamma \pi) \nu=2 \mu(\gamma D(u) \nu)_{\nu} \nu$, so that

$$
\gamma T(u, \pi) \nu=2 \mu \gamma D(u) \nu-(\gamma \pi) \nu=0 .
$$

Thus $(u, \pi)$ is a solution of $(N S E)_{1}$ on $J^{+}$.

Suppose that $(\tilde{u}, \tilde{\pi})$ is another such solution on $I$. Then the reductions, shown in Subsection 2.1, imply that $\tilde{u}$ is a $\mathbb{W}_{r}^{1}$-solution of $(C P)_{1}$ on $I$. By the uniqueness and maximality of $u$, it follows that $u \supset \tilde{u}$ and consequently that $\pi \supset \tilde{\pi}$. 
(b) Now assume that $k=2$. Again, we infer from Theorem 3.4 that there exists a unique maximal $\mathbb{W}_{r}^{1}$-solution of the reduced equations $(C P)_{2}$. Analogously as in (a) we see that $\boldsymbol{A}_{s / 2-1}=-\mu P_{2} \Delta$, so that

$$
\partial_{t} u-\mu P_{2} \Delta u=P_{2}(f-b(u)) \quad \text { in } L_{r, l o c}\left(J^{+}, H_{p, \mathcal{C}_{2}}^{s-2}\right) .
$$

We set

$$
\pi:=\left(K_{N} \gamma_{\nu}-R_{N} \operatorname{div}\right)(\mu \Delta u-b(u)+f) \in L_{r, l o c}\left(J^{+}, H_{p}^{s-1}(\Omega)\right),
$$

by formula (5). Then, thanks to $\nabla \pi=\left(i d-P_{2}\right)(\mu \Delta u-b(u)+f)$, we derive that

$$
\partial_{t} u+b(u)-\mu \Delta u+\nabla \pi=f \quad \text { in } L_{r, l o c}\left(J^{+}, H_{p}^{s-2}\right) .
$$

Hence, $(u, \pi)$ is indeed a solution of $(N S E)_{2}$ on $J^{+}$. We notice that the condition (38) is satisfied, due to the way in which we have defined the solution operators $K_{N}$ and $R_{N}$.

Finally let

$$
(\tilde{u}, \tilde{\pi}) \in \mathbb{W}_{r, \text { loc }}^{1}\left(I,\left(H_{p, \mathcal{C}_{2}}^{s}, H_{p, \mathcal{C}_{2}}^{s-2}\right)\right) \cap L_{r, l o c}\left(I, H_{p}^{s-1}(\Omega)\right)
$$

be another solution of $(N S E)_{2}$ on $I$. Then applying the Helmholtz projection $P_{2}$ to the first line of $(N S E)_{2}$ and using that $P_{2}(\nabla g)=0$ for every $g \in H^{s-1}(\Omega)$ yields

$$
\partial_{t} \tilde{u}-\mu P_{2} \Delta \tilde{u}=P_{2}(f-b(\tilde{u})) .
$$

Therefore, $\tilde{u}$ is a $\mathbb{W}_{r}^{1}\left(I,\left(H_{p, \mathcal{C}_{k}}^{s}, H_{p, \mathcal{C}_{k}}^{s-2}\right)\right)$-solution of $(C P)_{2}$ on $I$, and the assertion follows by the uniqueness and maximality of $u$.

Remarks 3.9. (a) In this article we have restricted ourselves to the boundary conditions

$$
\begin{aligned}
& \mathcal{T}_{1}(u, \pi):=\gamma T(u, \pi) \nu=2 \mu \gamma D(u) \nu-(\gamma \pi) \nu=0 \quad \text { and } \\
& \mathcal{T}_{2}(u, \pi):=(\gamma D(u) \nu)_{\tau}+(\gamma u)_{\nu} \nu=0 .
\end{aligned}
$$

However, if we supplement to Navier-Stokes equations with

$$
\begin{aligned}
& \mathcal{T}_{3}(u, \pi):=\mu \partial_{\nu} u-(\gamma \pi) \nu=0 \text { or } \\
& \mathcal{T}_{4}(u, \pi):=\left(\partial_{\nu} u\right)_{\tau}+(\gamma u)_{\nu} \nu=0,
\end{aligned}
$$

then, by obvious modifications of the proofs, all the results stated carry over to these problems. Indeed, the cases $k=3$ and $k=4$ are very similar to the stress-free and the no-slip problems, respectively.

(b) Let us consider the case $p=r$. In this situation Theorem 3.8 implies the existence of a unique maximal solution $(u, \pi)$ with

$$
u \in \mathbb{W}_{p}^{1}\left([0, T],\left(H_{p, \mathcal{C}_{k}}^{s}, H_{p, \mathcal{C}_{k}}^{s-2}\right)\right)
$$


for each $T \in \dot{J^{+}}$, provided

$$
\max \left\{-1+\frac{n+2}{p}, 1+\frac{1}{p}\right\}<s \leq 2 .
$$

and there exist $s_{0}, s_{1}>s$ such that

$$
\left(u^{0}, P_{k} f\right) \in F_{p, \mathcal{C}_{k}}^{s_{0}-2 / p} \times L_{p}\left(\mathrm{~J}, F_{p, \mathcal{C}_{k}}^{s_{1}-2}\right), \quad \operatorname{div}\left(u^{0}\right)=0 .
$$

(c) As already noted, the idea to reduce the Navier-Stokes equations $(N S E)_{k}$ to a parabolic pseudodifferential initial value problem is due to G. Grubb and V. A. Solonnikov [15], [16] and [17]. They prove existence and uniqueness results in anisotropic $L_{2}$ spaces (Bessel potential and Besov spaces). Later G. Grubb [14] extended these investigations to $L_{p}$ spaces. These results are difficult to compare to ours, since the regularities in time and space variables are linked together in anisotropic spaces (we refer to those papers for precise statements and definitions).

Under the same assumptions (42) and (43) Grubb, and Grubb and Solonnikov show existence and uniqueness of a solution $(u, \pi)$ with

$$
u \in H_{p}^{(s, s / 2)}(\Omega \times[0, T]) .
$$

(Actually, they can admit the limiting values $s_{0}=s_{1}=s=-1+(n+2) / p$ in many cases as well.) The solution spaces in (41) and (44) coincide (up to equivalent norms) if $s=2$. For $s<2$ they are, in general, not comparable. We can say, however, that functions in the anisotropic space in (44) possess less time regularity than those belonging to the space in (41).

\section{References}

[1] H. Amann, Linear and quasilinear parabolic problems, Vol. I: Abstract linear theory, Birkhäuser, Basel, Boston, Berlin, 1995.

[2] H. Amann, Compact embeddings of vector-valued Sobolev and Besov spaces, Glasnik Matematicki 35 (55) (2000).

[3] H. Amann, On the strong solvability of the Navier-Stokes equations, J. Math. Fluid Mech. 2 (2000), 16-98.

[4] H. Amann, Dual semigroups and second order linear elliptic boundary value problems, Israel J. Math. 45 (1983), 225-254.

[5] H. Amann, Dynamic theory of quasilinear parabolic equations - II, Reaction-diffusion systems, Diff. Int. Equ. 3 (1990), 13-75.

[6] H. Amann, Nonhomogeneous Navier-Stokes equations in spaces of low regularity, Topics in Mathematical Fluid Mechanics, Quaderni di Matematica 10 (2002), 13-31.

[7] H. Amann, Linear parabolic problems involving measures, RACSAM Rev. R. Acad. Cienc. Exactas Fis. Nat. Ser. A Mat. 95 (2001), 85-119.

[8] H. Amann, Nonhomogeneous Navier-Stokes equations with integrable low-regularity data, in: Nonlinear problems in mathematical physics and related topics, II. Int. Math. Ser., 1-28, Kluwer/Plenum, New York, 2002.

[9] E. B. Fabes, J. E. Lewis and N. M. Rivière, Boundary value problems for the NavierStokes equations, Amer. J. Math. 99 (1977), 628-668.

[10] Y. Giga, The nonstationary Navier-Stokes System with some first order boundary condition, Proc. Japan Acad. Ser. A 58 (1982), 101-104. 
[11] P. Grisvard, Équations différentielles abstraites, Ann. Sc. Éc. Norm. Sup. Sér. 4 (1969).

[12] G. GRubB, Functional calculus of pseudo-differential boundary problems, Progress in Math. Vol. 65, Birkhäuser, Boston, 1986.

[13] G. Grubb, Pseudo-differential boundary problems in $L_{p}$ spaces, Comm. P. D. E. 15 (1990), $289-340$.

[14] G. Grubb, Nonhomogeneous time-dependent Navier-Stokes equations in $L_{p}$ Sobolev spaces, Diff. Int. Equ. 8 (1995), 1013-1046.

[15] G. GrubB and V. A. Solonnikov, Reduction of fundamental initial-boundary value problems for the Stokes equation to parabolic initial-boundary value problems for systems of pseudodifferential equations, J. Soviet Math. 49 (1990), 1140-1147.

[16] G. Grubb and V. A. Solonnikov, Reduction of basic initial-boundary value problems for the Navier-Stokes equations to nonlinear parabolic systems of pseudodifferential equations, Zap. Nauchn. Sem. L.O.M.I. 171 (1989), 36-52.

[17] G. Grubb and V. A. Solonnikov, Boundary value problems for the Navier-Stokes equations treated by pseudo-differential methods, Math. Scand. 69 (1991), 217-290.

[18] J. Johnsen, The stationary Navier-Stokes equations in $L_{p}$-related spaces, Ph.D. thesis, Københavens Universitet, 1993.

[19] F. K. G. Odqvist, Über die Randwertaufgabeen der Hydrodynamik zäher Flüssigkeiten, Math. Z. 32 (1930), 329-376.

[20] R. T. Seeley, Interpolation in $L^{p}$ with boundary conditions, Stud. Math. 44 (1972), 47-60.

[21] Y. Shibita and S. Shimizu, On a resolvent estimate for the Stokes System with Neumann boundary condition, Diff. Int. Equ. 16 (4) (2003), 385-426.

[22] O. Steiger, On Navier-Stokes equations with first order boundary conditions, Ph.D. thesis, Universität Zürich, 2004.

[23] H. TRIEBEL, Interpolation theory, function spaces, differential operators, second ed., Johann Ambrosius Barth, Heidelberg, Leipzig, 1995.

Olivier Steiger

Institut für Mathematik

Universität Zürich

Winterthurerstrasse 190

CH-8057 Zürich

Switzerland

e-mail: osteiger@math.unizh.ch

(accepted: October 21, 2004; published Online First: October 27, 2005)

\section{(17) To access this journal online: (40) http://www.birkhauser.ch}

